\title{
Efficient Out-of-Distribution Detection Using Latent Space of $\beta$-VAE for Cyber-Physical Systems
}

\author{
SHREYAS RAMAKRISHNA, Vanderbilt University \\ ZAHRA RAHIMINASAB, Nanyang Technological University \\ GABOR KARSAI, Vanderbilt University \\ ARVIND EASWARAN, Nanyang Technological University \\ ABHISHEK DUBEY, Vanderbilt University
}

\begin{abstract}
Deep Neural Networks are actively being used in the design of autonomous Cyber-Physical Systems (CPSs). The advantage of these models is their ability to handle high-dimensional state-space and learn compact surrogate representations of the operational state spaces. However, the problem is that the sampled observations used for training the model may never cover the entire state space of the physical environment, and as a result, the system will likely operate in conditions that do not belong to the training distribution. These conditions that do not belong to training distribution are referred to as Out-of-Distribution (OOD). Detecting OOD conditions at runtime is critical for the safety of CPS. In addition, it is also desirable to identify the context or the feature(s) that are the source of OOD to select an appropriate control action to mitigate the consequences that may arise because of the OOD condition. In this article, we study this problem as a multilabeled time series OOD detection problem over images, where the OOD is defined both sequentially across short time windows (change points) as well as across the training data distribution. A common approach to solving this problem is the use of multi-chained one-class classifiers. However, this approach is expensive for CPSs that have limited computational resources and require short inference times. Our contribution is an approach to design and train a single $\beta$-Variational Autoencoder detector with a partially disentangled latent space sensitive to variations in image features. We use the feature sensitive latent variables in the latent space to detect OOD images and identify the most likely feature(s) responsible for the OOD. We demonstrate our approach using an Autonomous Vehicle in the CARLA simulator and a real-world automotive dataset called nuImages.
\end{abstract}

CCS Concepts: • Computer systems organization $\rightarrow$ Embedded and cyber-physical systems; $\bullet$ Artificial Intelligence and Cyber-Physical Systems;

Additional Key Words and Phrases: Cyber-physical systems, deep neural networks, out-of-distribution, disentanglement, $\beta$-variational autoencoders, mutual information gap

This work was supported by the DARPA Assured Autonomy program, the Air Force Research Laboratory, and in part by MoE, Singapore, Tier-2 grant number MOE2019-T2-2-040. Any opinions, findings, and conclusions, or recommendations expressed in this material are those of the author(s) and do not necessarily reflect the views of DARPA, AFRL, or MoE Singapore.

Authors' addresses: S. Ramakrishna, G. Karsai, and A. Dubey, Vanderbilt University, Nashville, Tennessee 37240; emails: \{shreyas.ramakrishna, gabor.karsai, abhishek.dubey\}@vanderbilt.edu; Z. Rahiminasab and A. Easwaran, Nanyang Technological University, 50 Nanyang Ave, Singapore 639798; emails: RAHI0004@e.ntu.edu.sg, arvinde@ntu.edu.sg.

Permission to make digital or hard copies of all or part of this work for personal or classroom use is granted without fee provided that copies are not made or distributed for profit or commercial advantage and that copies bear this notice and the full citation on the first page. Copyrights for components of this work owned by others than ACM must be honored. Abstracting with credit is permitted. To copy otherwise, or republish, to post on servers or to redistribute to lists, requires prior specific permission and/or a fee. Request permissions from permissions@acm.org.

(C) 2022 Association for Computing Machinery.

2378-962X/2022/04-ART15 \$15.00

https://doi.org/10.1145/3491243

ACM Transactions on Cyber-Physical Systems, Vol. 6, No. 2, Article 15. Publication date: April 2022. 
ACM Reference format:

Shreyas Ramakrishna, Zahra Rahiminasab, Gabor Karsai, Arvind Easwaran, and Abhishek Dubey. 2022. Efficient Out-of-Distribution Detection Using Latent Space of $\beta$-VAE for Cyber-Physical Systems. ACM Trans. Cyber-Phys. Syst. 6, 2, Article 15 (April 2022), 34 pages.

https://doi.org/10.1145/3491243

\section{INTRODUCTION}

Significant advances in Artificial Intelligence (AI) and Machine Learning (ML) are enabling dramatic, unprecedented capabilities in all spheres of human life, including Cyber-Physical Systems (CPSs). The fundamental advantage of AI methods is their ability to handle high-dimensional state-space and learn decision procedures or control algorithms from data rather than models. This is because high-dimensional real-world state spaces are complex and intractable for mathematical modeling and analysis. As such it is common to find AI components like Deep Neural Networks (DNNs) in real-world CPSs such as autonomous cars [6,56], autonomous underwater vehicles [17], and homecare robots [39]. However, there is still a gap in the safety and assurance of AI-driven CPSs as shown by well known incidents in recent past [40,72]. The technical debt is fundamentally in the black-box nature of AI components, which hinders the use of classical software testing strategies (e.g., code coverage, function coverage). There is research progress on testing [54, 68], however, wide-spread applicability remains questionable. The problem is exacerbated due to the way the systems are being designed.

The Safety Conundrum: CPS design flows focus on designing a system $S$ that satisfies some requirements $R$ in an environment $E$. During the design process, the developer selects component models, each including a parameter vector and typed ports representing the component interface, from a repository and defines an architectural instance ${ }^{1} A_{S}$ of the system such that $A_{S} \| E \mid=R$, while satisfying any compositional constraints across the component boundaries, often specified as pre-conditions and post-conditions [15]. The difficulty in this process is that in practice the environment is only approximated using a surrogate model $\hat{E}$ or a set of observations $|\tilde{\hat{E}}|$ collected from real-world data. It is clear that $A_{S} \| \tilde{\hat{E}}=R$ does not imply $A_{S} \| E \mid=R$. In this sense, the system is being deployed with the assumption that $\tilde{\hat{E}} \approx E$. However, this is not a strong guarantee and could result in scenarios where the designed architecture may fail in the physical environment. Hence, runtime monitoring of the system is required to identify when $\tilde{\hat{E}} \not E$ i.e., the observed samples are Out-of-Distribution (OOD) with the real environment.

To contextualize the problem, consider the case of a perception DNN that consumes a stream of camera images to predict control actions (e.g., steer and speed) for autonomous driving tasks such as end-to-end driving [6]. In this context, the stream of images can be categorized as scenes. A scene is short time series of similar images contextualized by certain environmental features such as weather, brightness, road conditions, traffic density, among others, as shown in Figure 1. These features are referred to as semantic labels [52] or generative factors [29], and in this article, we refer to them as features. These features can take continuous or discrete values, and these values can be sampled for generating different scenes as shown in Figure 1. The images from these scenes are collectively used for training the DNN. These features effectively specify the context in which the system is operating and influence the sensitivity and correctness of the DNN's predictions, especially in cases where the features representing the scenes used for training do not cover all

\footnotetext{
${ }^{1} \mathrm{An}$ architectural instance of a design is a labeled graph where nodes are the ports of the components, and the edges represent interactions between the ports.
} 


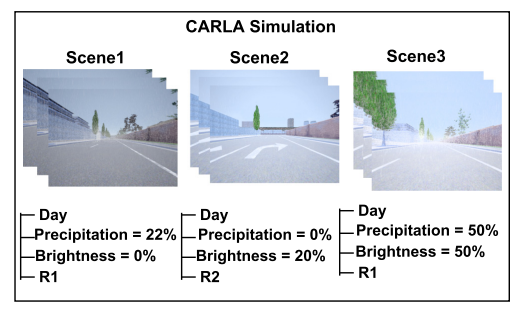

(a)

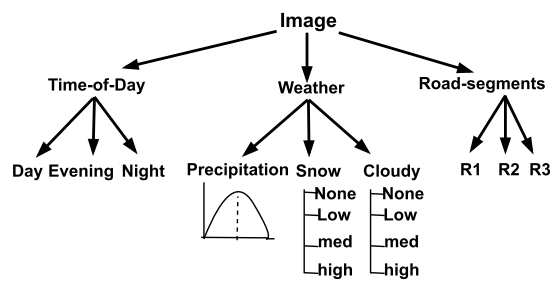

(b)

Fig. 1. Scenes and feature representations. (a) scenes with feature labels from CARLA simulation, (b) hierarchical representation of an image using its features. The features can take discrete or continuous values.

the values found in real-world. In this work, we primarily focus on perception DNNs, so we define the problem in terms of images.

Problem Definition: The problem, in this case, is to identify: (a) if the current image of the operational scene is OOD with respect to the training set, and (b) feature(s) likely responsible for the OOD. By this, we mean that if the training set used to train a DNN did not include the scenes with heavy rain, then we want to identify during operation that the OOD is due to precipitation. In addition, as the images received by CPSs are in time series, it is important to identify if the current image has changed with respect to the previous images in the time series. Identifying these changes is referred to as change point detection in literature. Change points in the values of a feature can increase the system's risk, as illustrated in our previous work [26]. So, it is critical to identify these change points during operation. Formally, we summarize the problems as follows: Problem $1 a$ - identify if the current image is OOD with respect to the training set. Problem $1 b$ identify the feature(s) most likely responsible for the OOD, and Problem 2 - identify if the current image is OOD to the previous images in time series.

State-of-the-art: Multi-chained one-class classifiers [70] are commonly used for solving the multi-label anomaly detection problem. But the performance of these chains deteriorates in the presence of strong label correlation [80]. Additionally, training one classifier for each label gets expensive for real-world datasets which have a large label set [58]. Another problem with traditional classifiers like Principal Component Analysis [60], Support Vector Machine [63], and Support Vector Data Description (SVDD) [77] is that they fail in images due to computational scalability [62].

To improve the effectiveness of the classifiers, researchers have started investigating probabilistic classifiers like Generative Adversarial Network (GAN) [23] and Variational Autoencoder (VAE) [36]. GAN has emerged as the leading paradigm in performing unsupervised and semisupervised OOD detection [1,81], but problems of training instability and mode collapse [10, 75] have resulted in VAE based methods being used instead. In particular, the VAE based reconstruction approach has become popular for detecting OOD data [2, 8]. However, this approach is less robust in detecting anomalous data that lie on the boundary of the training distribution [12]. To address this, the latent space generated by a VAE is being explored [12, 66, 71]. The latent space is a collection of latent variables $(L)$, where each latent variable is a tuple defined by the parameters $(\mu, \sigma)$ of a latent distribution $(z)$ and a sample generated from the distribution. However, the traditional approach of just training a single VAE on all input data leads to unstructured and entangled distributions [38], which makes the task of isolating the feature(s) responsible for OOD hard.

This article: Our approach in this work is to investigate use of latent space disentanglement for detecting OOD images used by perception DNNs. This idea builds upon recent progress in structuring and disentangling the latent space [5, 29]. Effectively, the latent space generated by the encoder of a VAE is a Gaussian mixture model of several overlapping and entangled latent 

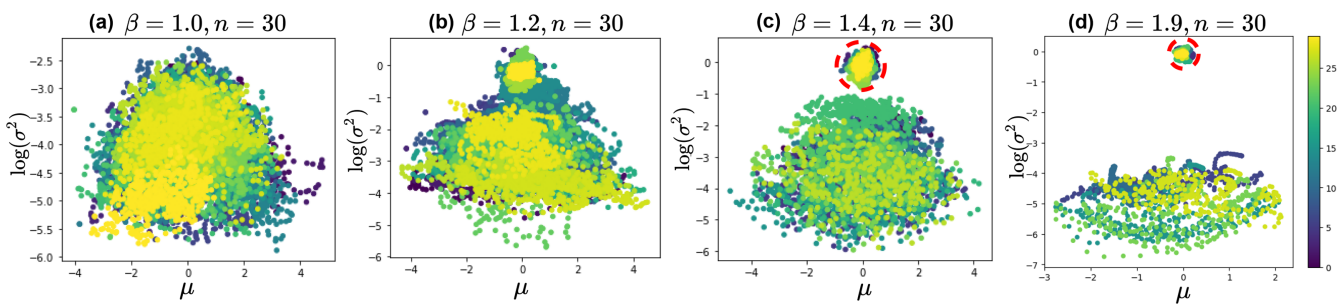

Fig. 2. Visualizing latent space disentanglement. Scatter plots illustrating the latent distributions $\left(\mu, \log \left(\sigma^{2}\right)\right)$ generated using a $\beta$-VAE with different $\beta$ values. Each latent distribution in the latent space is represented using distinct color shades. CARLA images generated in Section 5 was used to generate these latent distributions. For $\beta=1$, the generated latent distributions are entangled. For $\beta>1$, the latent distributions are partially disentangled with a few latent distributions (inside the red circle) encoding independent features moving close to $\mu=0$ and $\log \left(\sigma^{2}\right)=0$, and the others moving away. Plot axis: $x$-axis represents the mean of the latent distributions in the range $[-5,5]$, and the $y$-axis represents the log of variance in the range $[-5,5]$.

variables, each of which encodes information about the image features. However, as can be seen in Figure 2(a), the latent variables form a single large cluster which makes it hard to use them for OOD detection. Disentanglement is a state of the latent space where each latent variable is sensitive to changes in only one feature while being invariant to changes in the others [5]. That is, the single large cluster of Figure 2(a) is separated into several smaller clusters of single latent variables if the features are independent. Such disentangled latent variables have been successfully used in several tasks like face recognition [55, 69], video predictions [30], and anomaly detection [76]. However, disentangling all the latent variables is extremely hard for real-world datasets and is shown to be highly dependent on inductive biases [44] and feature correlations.

Nevertheless, even partially disentangling the latent variables can lead to substantial gains as shown by Jakab et al. [32] and Mathieu et al. [49]. Partial disentanglement is a heuristic that groups the most informative latent variables into one cluster and the remaining latent variables that are less informative into another, as shown in Figure 2(c). This selective grouping enables better separation in the latent space for the train and test images as shown in Figure 10 (see Section 5). However, the procedure for training a disentangled latent space for real-world CPSs is hard. As a result, it is one of the aspects we focus on in this article, along with interpreting the source of the OOD.

Our Contributions: We present an approach to generate a partially disentangled latent space and learn an approximate mapping between the latent variables and the image features to perform OOD detection and reasoning. The steps in our approach are data partitioning, latent space encoding, latent variable mapping, and runtime anomaly detection. To generate the partially disentangled latent space, we use a $\beta$-Variational Autoencoder $(\beta$-VAE), which has a gating parameter $\beta$ that can be tuned to control information flow between the features and the latent space. For a specific combination of $\beta>1$ and the number of latent variables $(n)$, the latent space gets partially disentangled with few latent variables encoding most of the feature's information, while the others, encoding little information. We present a Bayesian Optimization heuristic to find the appropriate combination of the $\beta$ and $n$ hyperparameters. The heuristic establishes disentanglement as a problem of tuning the two hyperparameters. The selected hyperparameters are used to train a $\beta$-VAE that is used by a latent variable mapping heuristic to select a set of most informative latent variables that are used for detection and identify the sensitivity of the latent variables towards specific features. The sensitivity information is used for reasoning the OOD images. Finally, the trained $\beta$-VAE along with the selected latent variables are used at runtime for OOD detection and reasoning. We demonstrate our approach on two CPS case studies: (a) an Autonomous Vehicle (AV) in CARLA simulator [14], and (b) a real-world automotive dataset called nuImages [52]. 


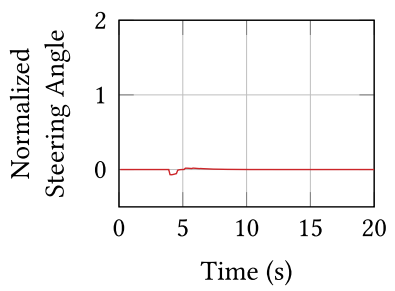

(a)

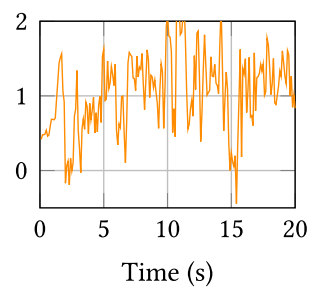

(b)

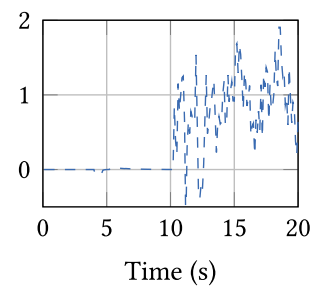

(c)

Fig. 3. Problem illustration. We trained an NVIDIA DAVE-II DNN with images from scene 1 and scene2 (Figure 1) to predict the steering values of an AV that travels on a straight road segment in CARLA simulator. We tested the network on three test scenes: (a) A scene that had precipitation and brightness values within the training distribution, (b) A scene with high precipitation (60\%) that was not in the training distribution. For this scene, the DNN predictions deviate from the nominal value shown in the plot a, and (c) A scene where brightness value changed from low (20\%) to high (50\%) at $t=10$ seconds. The DNN predictions were accurate until $t=10$ seconds, thereafter the predictions got erroneous.

Outline: The outline of this article is as follows. We formulate the OOD detection problem in Section 2. We introduce the background concepts in Section 3. We present our OOD detection approach in Section 4. We discuss our experiment setup and evaluation results in Section 5. Finally, we present related research in Section 6 followed by conclusions in Section 7.

\section{PROBLEM FORMULATION}

To set up the problem, consider a CPS that uses a perception DNN trained on image distribution $\mathcal{T}$, where $\mathcal{T}=\left\{s_{1}, s_{2}, \ldots, s_{i}\right\}$ is a collection of scenes. A scene is a collection of sequential images $\left\{I_{1}, I_{2}, \ldots, I_{m}\right\}$ generated from a training distribution $P(\mathcal{T})$. Every image in a scene is associated with a set of discrete or continuous valued labels $\left(I \rightarrow 2^{\mathbb{L}}\right)$ belonging to the generative features of the environment (see Figure 1). It is important to note that the sampling rate of images depends on the dynamics of the system. With this model, we can define the problems we study as follows:

Problem 1. Given a test image $I_{t}$, determine (a) if $I_{t} \in P(\mathcal{T})$, and $(\boldsymbol{b})$ if $\left(I_{t} \notin P(\mathcal{T})\right)$ then identify the feature $f$ whose $\operatorname{Label}\left(I_{t}, f\right) \notin P_{f}(\mathcal{T})$, where $P_{f}$ is the training distribution on the feature $f$.

Example. To illustrate the problem, we trained an NVIDIA DAVE-II DNN [6] to perform e2e driving of an AV in CARLA simulation. We trained the network on camera images from scene1 and scene2 (see Figure 1) to predict the steering control action for the AV. As shown in Figure 3(a), the network's steering predictions were accurate when tested on images from training scenes. However, the predictions got erroneous when we used the network to predict on images of a new scene (scene3) with higher precipitation values outside the training distribution (Figure 3(b)). The error in steering predictions caused the AV crash of a sidewall. For this reason, if we knew that the precipitation level is compromising the network's predictions, we can switch to an alternative controller that operates on other sensor inputs (e.g., Radar or Lidar) rather than the camera images.

Problem 2. Given the test image $I_{t}$ at time $t$, the goal is to determine if $I_{t}$ has changed with respect to the previous images in a time series window $(I[t-M+1], \ldots, I[t])$, where $M$ is the window size.

Example. To illustrate the problem, we use the same AV setup discussed in problem 1 . We tested the trained network on a new scene (scene4) with low brightness (in-distribution) for up to 10 seconds, and the brightness was briefly high (OOD) for the next 10 seconds. For this scene, the network predicted accurately for the first 10 seconds and erroneously for the next 10 seconds as 
shown in Figure 3(c). Such an abrupt change in the image feature increases the AV's risk of collision as demonstrated in our recent work [26]. So, identifying the change points can be beneficial for reducing the system's risk of a consequence.

Detector Requirements: We evaluate OOD detectors that solve these problems against the following properties.

- Robustness - The detector should have low false positives and false negatives. A well known metric to measure robustness is F1-score $=(2 \cdot$ Precision $\cdot$ Recall $) \div($ Precision + Recall $)$. Precision is computed as $T P \div(T P+F N)$, and Recall is computed as $T P \div(T P+F P)$. Where TP is True positive, FP is False positive, and FN is false negative.

- Minimum Sensitivity (MS) - The detector should have a minimum sensitivity towards each $\overline{\text { feature [48]. For an image }} I_{t}$ with feature labels $\mathbb{L}=\left\{l_{1}, l_{2}, \ldots l_{k}\right\}$, minimum sensitivity is defined as the minimum value of the detector's sensitivity for each feature label. $M S=$ $\min \{S i ; i=1,2, \ldots, k\}$, where $S_{i}$ is the sensitivity of the detector to the feature $i$. Recall has been a well known metric to measure the detector's sensitivity.

- Low Computation Overhead - Our target platforms are resource constrained autonomous CPSs like DeepNNCar [57]. Therefore, the detector should have a low resource signature.

- Low Execution time - Autonomous CPSs typically have a small sampling period (typically 50 to 100 milliseconds). Therefore, the detector should have a low execution time that is smaller than the system's sampling period.

\section{BACKGROUND}

In this section, we provide an overview of several basic concepts that are required to understand our OOD detection approach.

\subsection{Kullback-Leibler (KL) Divergence}

KL-divergence [16] is a non-symmetric metric that can be used to measure the similarity between two distributions. For any probability distribution $p$ and $q$ the KL-divergence can be computed as illustrated in Equation (1). A KL-divergence value close to zero indicates the two distributions are similar, while a larger value indicates their dissimilarity.

$$
D_{K L}(p \| q)=\sum_{x \in \chi} p(x) \log \frac{p(x)}{q(x)} .
$$

Recently, the KL-divergence metric is being utilized in several ways: (a) training loss function of different generative models (e.g., VAE) called the Evidential Lower Bound (ELBO) [37], and (b) OOD detection metric [71] that measures if the latent distributions generated by a generative model deviate from a standard normal distribution. The metric can be computed as shown below.

$$
K L(x)=D_{K L}\left(q_{\phi}\left(z_{i} \mid x\right) \| \mathcal{N}(0,1)\right),
$$

where, $\mathcal{N}(0,1)$ is a standard normal distribution. $q_{\phi}\left(z_{i} \mid x\right)$ is the distribution generated by the encoder of a VAE for a latent variable $L_{i}$, and input $x$.

\section{$3.2 \beta$-Variational Autoencoder $(\beta$-VAE)}

$\beta$-Variational Autoencoder [29] is a variant of the original VAE with a $\beta$ hyperparameter attached to the KL-divergence (second term) of the ELBO loss function shown in Equation (3). The network has an encoder that maps the input data $(x)$ distribution $P(x)$ to a latent space $(z)$ by learning a posterior distribution $q_{\phi}(z \mid x)$. The decoder then reconstructs a copy of the input data $\left(x^{\prime}\right)$ by sampling the learned distributions of the latent space. In doing so, the decoder also learns a likelihood 
distribution $p_{\theta}(x \mid z)$. The latent space is a collection of $n$ latent variables that needs to be selectively tuned in accordance with the input dataset. To remind, a latent variable is a tuple defined by the parameters $(\mu, \sigma)$ of a latent distribution $(z)$ and a sample generated from the distribution.

$$
\operatorname{ELBO}(\theta, \phi, \beta ; x, z)=\mathbb{E}_{q_{\phi}(z \mid x)}\left[\log p_{\theta}(x \mid z)\right]-\beta D_{K L}\left(q_{\phi}(z \mid x) \| p(z)\right) .
$$

$\theta$ and $\phi$ parameterize the latent variables of the encoder and the decoder, and $D_{K L}$ is the KL-divergence metric discussed in Section 3.1. The first term computes the similarity between the input data $x$ and the reconstructed data $x^{\prime}$. The second term computes the KL-divergence between $q_{\phi}(z \mid x)$ and a pre-defined distribution $p_{\theta}(x \mid z)$, which is mostly the standard normal distribution $\mathcal{N}(0,1)$.

Tuning $\beta$ for disentanglement: As suggested by Higgins et al. [29], $\beta$ controls the amount of information that flows from the features to the latent space, and an appropriate hyperparameter combination of $\beta>1$ and $n$ is shown to disentangle the latent space for independent features. Figure 2 shows the latent space disentanglement for different values of $\beta$ while $n$ is fixed to 30 . For $\beta=1$, the latent distributions are entangled. With $\beta>1$, the latent space starts to partially disentangle with a few latent variables (inside the red circle) encoding most information about the features stay as a cluster close to $\mu=0$ and $\log \left(\sigma^{2}\right)=0$, and the others are uninformative and form a cluster that lies farther. However, as the $\beta$ value gets larger $(\beta=1.9)$, the information flow gets so stringent that the latent space becomes uninformative [49]. So, finding an appropriate combination of $\beta$ and $n$ for disentanglement is a hard problem. To address this problem, we provide a heuristic in Section 4.2.

\subsection{Mutual Information Gap (MIG)}

Mutual Information Gap is a metric proposed by Chen, Ricky TQ et al. [9] to measure the latent space disentanglement. It is an information theoretic metric that measures the mutual information between features and the latent variables. It measures the average difference between the empirical mutual information of the two most informative latent variables for each feature and normalizes this result by the entropy of the feature. MIG is computed using the following equation.

$$
M I G=\frac{1}{|\mathcal{F}|} \sum_{f \in \mathcal{F}} \frac{1}{H(f)}\left(I_{1}\left(L_{j} ; f\right)-I_{2}\left(L_{j}^{\prime} ; f\right)\right) .
$$

$I_{1}\left(L_{j} ; f\right)$ represents the empirical mutual information between the most informative latent variable $L_{j}$ and the feature $f . I_{2}\left(L_{j}^{\prime} ; f\right)$ represents the empirical mutual information between the second most informative latent variable $L_{j}^{\prime}$ and the feature $f . H(f)$ is the entropy of information contained towards the feature $f$. In this work, we use MIG as a measure for selecting the right hyperparameter combination $(\beta$ and $n)$ for the $\beta$-VAE.

Implementation: We have implemented Algorithm 1 to compute MIG. Since MIG is computed based on entropy, the training set $\mathcal{T}$ should have images with feature labels that take different discrete and continuous values as shown in Figure 1. For the ease of computation, we partition $\mathcal{T}$ into different partitions $\mathcal{P}$ using our approach discussed in Section 4.1. Our approach is to create partitions such that each partition will have images that have a variance in the value of a specific feature $f$, irrespective of the variance in the others. The feature with the highest variance represents the partition. Then, in each iteration, we use the feature representing the partition to compute the feature entropy, the conditional entropy, and the mutual information of the two most informative latent variables. The mutual information is then used to compute the MIG as shown in the algorithm. Finally, for robustness, we average the MIG across $t$ iterations.

Complexity Analysis: The complexity of the MIG algorithm in worst case is $O(t *|\mathcal{P}| * n q *|\mathcal{F}| *$ $\left.|\mathcal{T}| * n^{2} * n s\right)$. Where $t$ is the number of iterations, $|\mathcal{P}|$ is the number of partitions, $n$ is the number 


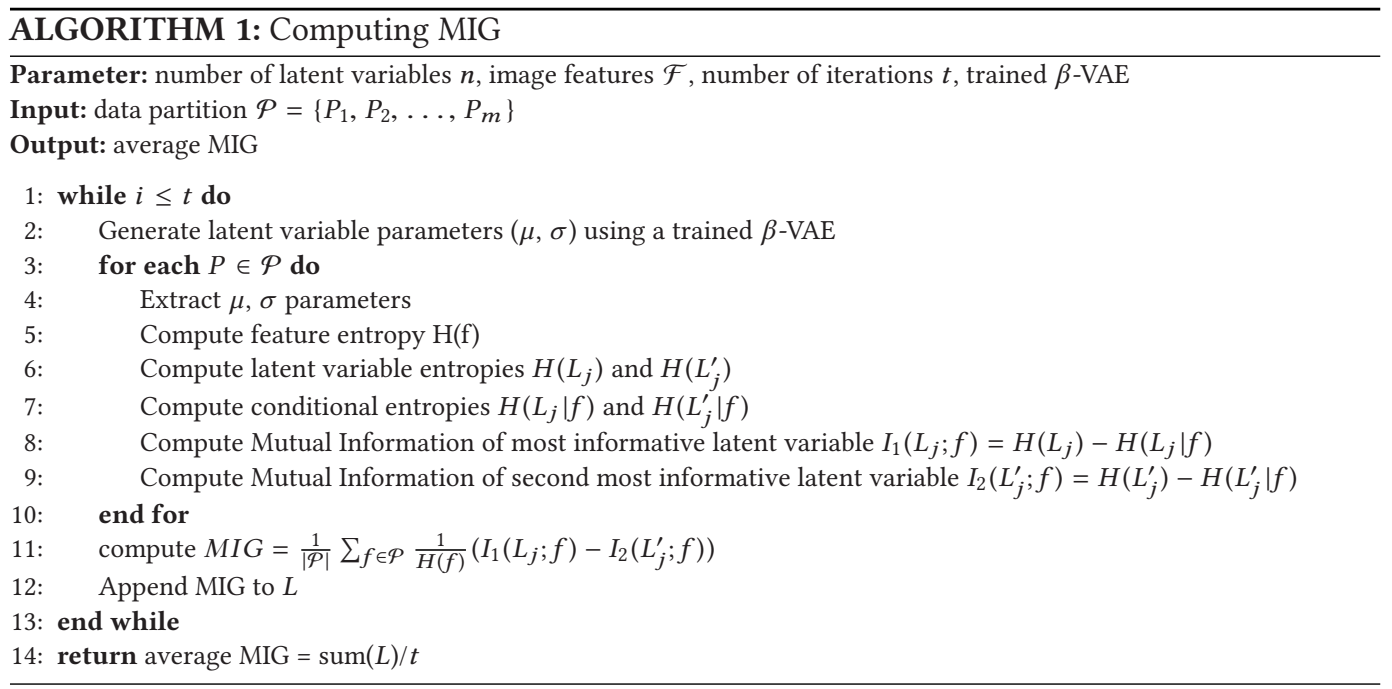

of latent variables, $|\mathcal{F}|$ is the number of features considered for the calculations, $n q$ is the number of unique values that each feature has in the partition (e.g., for a partition with brightness $=10 \%$, and brightness $=20 \%, n q=2)$, $n s$ is the number of samples generated from each latent variable, and $|\mathcal{T}|$ is the size of training data. The specific values of these parameters for the AV example in CARLA simulation are $t=5,|p|=2, n=30, \mathcal{F}=2, n s=500$, and $n q=3$.

\subsection{Inductive Conformal Prediction (ICP)}

Inductive Conformal Prediction is a variant of the Conformal Prediction algorithm [74] that tests if a test observation $\left(x_{t}\right)$ conforms to every observation in the training dataset $(\mathcal{T})$. However, comparing $x_{t}$ to every observation of $\mathcal{T}$ is expensive and gets complex with the size of $\mathcal{T}$. To address this, ICP splits $\mathcal{T}$ into two non-overlapping sets called as the proper training set $\left(\mathcal{T}_{P}\right)$ which is used to train the prediction algorithm (e.g., DNN) and the calibration set $(C)$ which is used to calibrate the test observations. In splitting the datasets, ICP performs a comparison of the $x_{t}$ to each element in the $C$ which is a smaller representative set of $\mathcal{T}$. The ICP algorithm has two steps: The first step involves, computing the non-conformity measure, which represents the dissimilarity between $x_{t}$ to the elements in the set $C$. The non-conformity measure is usually computed using conventional metrics like euclidean distance or $K$-nearest neighbors. But, in this work, we use KL-divergence as the non-conformity measure. The next step involves computing the $p$-value, which serves as evidence for the hypothesis that $x_{t}$ conforms to $C$. Mathematically, the $p$-value is computed as the fraction of the observations in $C$ that have non-conformity measure above the test observation $x_{t}$ : $p_{x_{t}}=\left|\left\{\forall \alpha \in C \mid \alpha \geq \alpha_{x_{t}}\right\}\right| /|C|$. Note for brevity, we drop the notation of $x$ and just use the term $p_{t}$ and $\alpha_{t}$. Here $\alpha$ denotes the non-conformity measure for each observation in $C$ and $\alpha_{t}$ denotes the non-conformity measure for $x_{t}$.

Once $p_{t}$ is computed, it can be compared against a threshold $\tau \in(0,1)$ to confirm if $x_{t}$ belongs to $\mathcal{T}$. However, such a threshold-based comparison is only valid if each test observation is i.i.d (independent and identically distributed) to $\mathcal{T}$, which is not true for CPSs [8]. Although the assumptions about i.i.d are not valid, ICP can still be applied under the weaker assumption of exchangeability. In our context, exchangeability means to test if the observations in $C$ have the same joint probability distribution as the sequence of the test observations under consideration (are they permutations of each other). If they are, then we can expect the $p$-values to be independent and uniformly 
distributed in $[0,1]$ (Theorem 8.2, [74]), which can be tested using the martingale. Exchangeability martingale [18] has been used as a popular tool for testing the exchangeability and the i.i.d assumptions of the test observation with respect to $C$. So, once the $p$-value for a test observation is computed, the simple mixture martingale [18] can be computed as $\mathcal{M}_{t}=\int_{0}^{1}\left[\prod_{i=1}^{t} \epsilon p_{i}^{\epsilon-1}\right] d \epsilon$.

Also, it is desirable to use a sequence of test observations rather than a single observation for improving the detection robustness. However, the observations received by CPS are in time series, which makes them non-exchangeable [8]. The non-exchangeability nature hinders the direct application of the martingale to an infinitely long sequence of test observations. To address this, the authors in [8] have suggested applying the martingale over a short window of the time series in which the test observations can be assumed to be exchangeable. Then, the simple mixture martingale over a short time window $[t-M+1, t]$ of past $M p$-values can be computed as $\mathcal{M}_{t}=\int_{0}^{1}\left[\prod_{i=t-M+1}^{t} \epsilon p_{i}^{\epsilon-1}\right] d \epsilon$. The martingale will grow over time if and only if there are consistently low $p$-values within the time window, and the corresponding test observations are i.i.d. Otherwise, the martingale will not grow. It is important to note that the martingale computation is only valid for a short time window. The size of the window is dependent on the CPS dynamics, like the speed of the system. In our experiments, the system's speed was constant, so we used a fixed window size of 20 images.

\subsection{Cumulative Sum (CUSUM)}

One of the problems we are dealing with is change detection. This problem is traditionally solved using CUSUM [3], which is a statistical quality control procedure used to identify variation based on historical data. It is computed as $S_{0}=0$ and $S_{t+1}=\max \left(0, S_{t}+x_{t}-\omega\right)$, where $x_{t}$ is the sample from a process, $\omega$ is the weight assigned to prevent $S_{t}$ from consistently increasing to a large value. $S_{t}$ can be compared to a pre-defined threshold $\tau$ to perform the detection. $\omega$ and $\tau$ are hyperparameters that decide the detector's precision.

\section{OUR APPROACH}

In this section, we present our approach that uses a $\beta$-VAE to perform latent space based OOD detection and reasoning. Our approach is shown in Figure 4 and the steps involved work as follows: First, we divide the multi-labeled datasets into partitions based on the variance in the feature values. A partition consists of images with one feature having higher variance in its values compared to others. Second, we generate a partially disentangled latent space using a $\beta$-VAE. As discussed earlier, the combination of $\beta$ and $n$ influence the level of latent space interpretability. To find the optimal combination, we propose a heuristic that uses the Bayesian Optimization algorithm [65] along with MIG to measure disentanglement. Third, we discuss a heuristic to perform latent variable mapping to identify the set of latent variables $\mathcal{L}_{d}$ that encodes most information of the image features. These latent variables are collectively used as the detector for OOD detection. Further, we perform a KL-divergence based sensitivity analysis to identify the latent variable(s) $\mathcal{L}_{f} \subseteq \mathcal{L}_{d}$, that is sensitive to specific features. The latent variable(s) in $\mathcal{L}_{f}$ are used as reasoners to identify the OOD causing features. We discuss these steps in the rest of this section.

\subsection{Data Partitioning}

Data partitioning is one of the core steps of our approach. It is required for implementing the MIG and the latent variable mapping heuristics. We define a partition $P$ as a collection of images that have higher variance in the value of one feature as compared to the variances in the values of other features. To explain the concept of a partition, consider the features of images in training set $\mathcal{T}$ to be $\mathcal{F}=\left\{f_{1}, f_{2}, \ldots, f_{n}\right\}$, and each feature can take a value either discrete or continuous as shown 


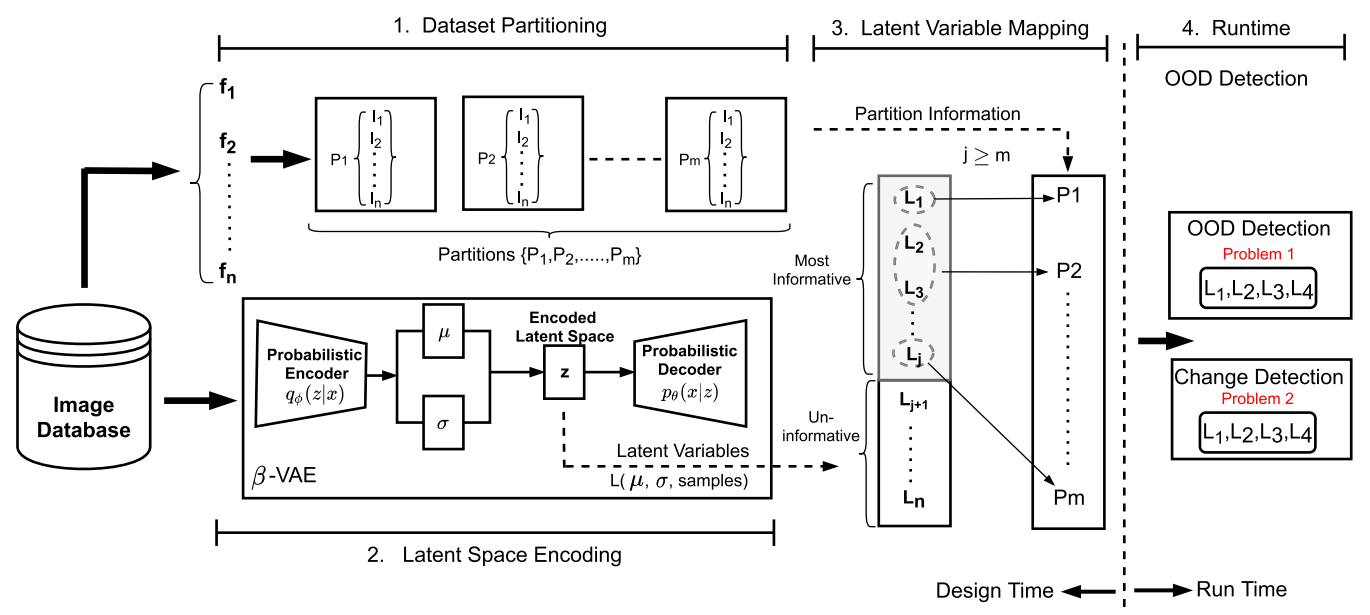

Fig. 4. Detector design approach. The steps of our approach include data partitioning, latent space encoding, latent variable mapping, and runtime OOD detection.

in Figure 1. We normalize these values for the ease of partitioning. Our goal is to group images in $\mathcal{T}$ into $m$ partitions $\mathcal{P}=\left\{P_{1}, P_{2}, \ldots, P_{m}\right\}$, such that a partition $P_{j}$ will have all the images with high variance in the values of feature $f_{j}$. For creating these partitions, we generate sub-clusters for each discrete valued feature(s) and sub-clusters for each continuous valued feature. In each of these sub-clusters, only the value of the feature under consideration $f_{j}$ changes while the value of other features remains unchanged. The clustering is done effectively through an agglomerative clustering algorithm. Thereafter, the partition for a feature is the union of all sub-clusters which can be represented as $P_{j}=\left\{C_{1} \cup C_{2} \cdots \cup C_{n}\right\}$. It is important to note that each partition should have variance $>0$ in the value of the feature under consideration $\left(f_{j}\right)$, irrespective of changes in the values of other features. To illustrate the partitioning concept, consider the example scenes in Figure 1. A precipitation partition for this example is a collection of images from two combinations, $C 1=\{$ day, precipitation $=22 \%$, brightness $=0 \%, \mathrm{R} 1\}$, and $C 2=$ day, precipitation $=50 \%$, brightness $=50 \%, \mathrm{R} 1\}$.

Our data partitioning approach works well for datasets with well defined labels that provide feature value(s). However, real-world automotive datasets such as nuScenes [7] and nuImages [52] provide semantic labels that are not always well defined, and they do not contain feature values. Also, they often have images in which several feature values change at once. Since the feature related information is not fully available, some pre-possessing and thresholds selection for feature values are required for partitioning. Currently, the threshold selection for partitioning is performed by a human supervisor, but we want to automate it in the future. We have applied this partitioning technique on the nuScenes dataset in our previous work [66] and used it in this work for partitioning the nuImages dataset.

\subsection{Latent Space Encoding}

The second step of our design procedure is the selection and training of $\beta$-VAE to generate a partially disentangled latent space encoding. However, the challenge is to determine the best combination of the $\beta$ and $n$ hyperparameters. To find this, we propose a novel greedy heuristic that formulates disentanglement as a hyperparameter search problem. The heuristic uses Bayesian Optimization (BO) algorithm with MIG as the objective function to maximize. 


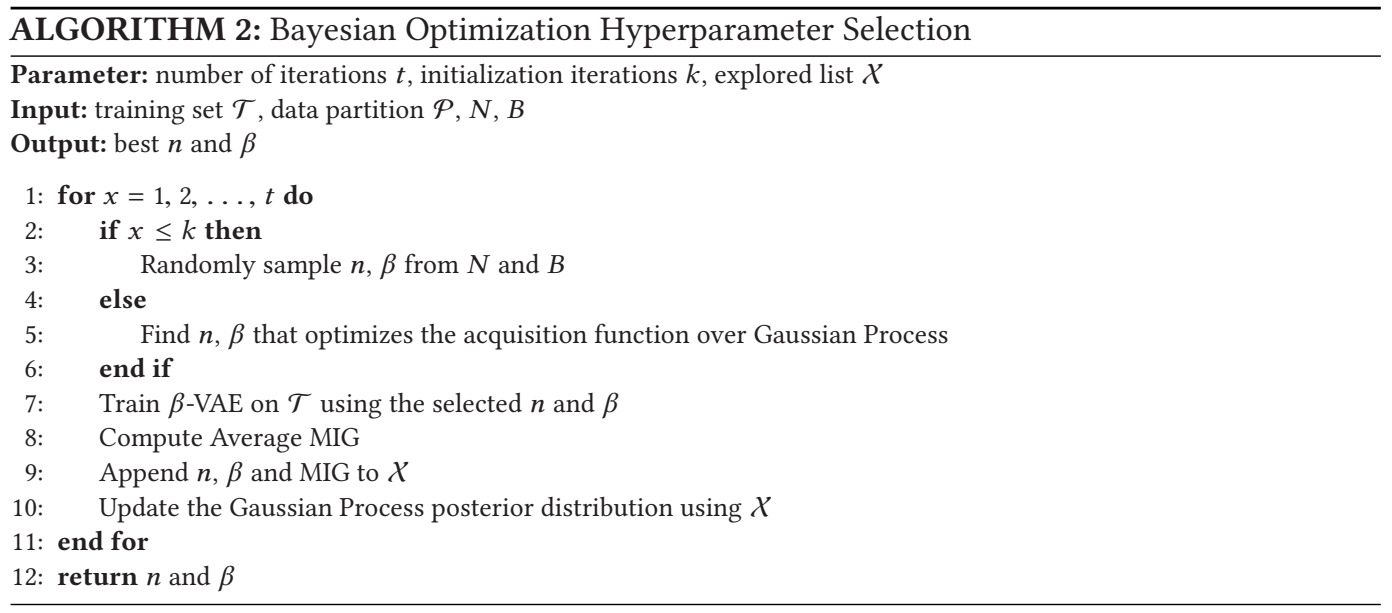

Implementation: The $\mathrm{BO}$ algorithm builds a probability model of the objective function and uses it to identify the optimal model hyperparameter(s). The algorithm has two steps: a probabilistic Gaussian Process model that is fitted across all the hyperparameter points that are explored so far, and an acquisition function to determine which hyperparameter point to evaluate next [65]. We use these steps to search for an optimal hyperparameter for $\mathrm{n} \in N$, and $\beta \in B$. The heuristic using BO algorithm is shown in Algorithm 2, and the steps are discussed below.

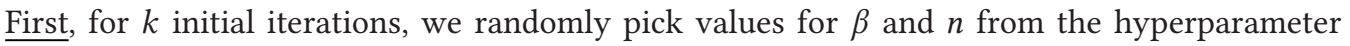
search space. The randomly selected $\mathrm{n}$ and $\beta$ combination is used to train a $\beta$-VAE network and compute the MIG as discussed in Algorithm 1. The selected hyperparameters and the computed MIG are added to an explored list $\mathcal{X}$. After the initial iterations, the trained Gaussian Process model (the initial iterations are used to train and stabilizes the Gaussian process model) is fitted across all the hyperparameter points that were previously explored, and the marginalization property of the Gaussian distribution allows the calculation of a new posterior distribution $\mathrm{g}\left(x_{n}\right)$ with posterior belief $\tilde{g}\left(x_{n}\right)$. Finally, the parameters $(\mu, \sigma)$ of the resulting distribution are determined to be used by the acquisition function, which uses the posterior distribution to evaluate new candidate hyperparameter points.

Second, an acquisition function is used to guide the search by selecting the hyperparameter(s) for next iteration. For this, it uses the $\mu$ and $\sigma$ computed by the Gaussian process. A commonly used function is the expected improvement (EI) [21, 34], which can described as follows. Consider, $x_{n}$ to be some hyperparameter(s) point in the distribution $\mathrm{g}\left(x_{n}\right)$ with posterior belief $\tilde{g}\left(x_{n}\right)$, and $x_{+}$is the best hyperparameter(s) in $\mathcal{X}$ (explored list), then the improvement of the point $x_{n}$ is computed against $x_{+}$as $I\left(x_{n}\right)=\max \left\{0,\left(g\left(x_{+}\right)-g\left(x_{n}\right)\right)\right\}$. Then, the expected improvement is computed as $E I\left(x_{n}\right)=\mathbb{E}\left[I\left(x_{n}\right) \mid x_{n}\right]$. Finally, the new hyperparameter(s) is computed as the point with the largest expected improvement as $x_{n+1}=\operatorname{argmax}\left(E I\left(x_{n}\right)\right)$. The new hyperparameter point is used to train a $\beta$-VAE and compute the MIG, which is then added to the explored list $\mathcal{X}$. The list $\mathcal{X}$ is used to update the posterior distribution of the Gaussian process model in the next iteration. The two steps of the algorithm are iterated until maximum number of iterations is reached or can be terminated early if optimal hyperparameter(s) is consecutively selected by the algorithm for $j$ iterations (we chose $j=3$ in this work).

Design Space Complexity: Searching for optimal hyperparameters is a combinatorial problem that requires optimizing an objective function over a combination of hyperparameters. 


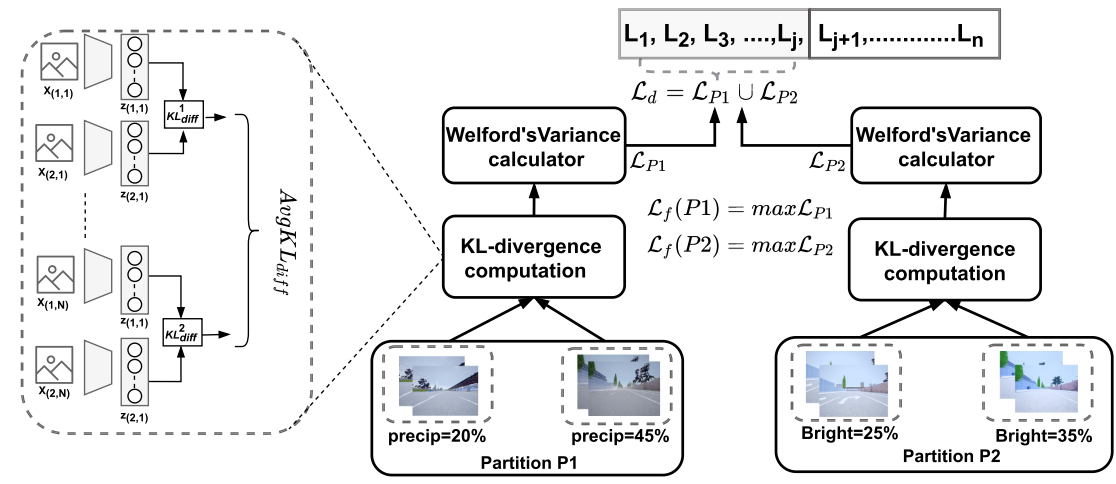

Fig. 5. Latent variable mapping. Heuristic based on KL-divergence and Welford's variance calculator to select latent variables for $\mathcal{L}_{d}$ and $\mathcal{L}_{f}$.

In our context, the $\beta$-VAE hyperparameters to be selected are $\beta$ and $n$, and the objective function to optimize is the MIG whose complexity we have reported in the previous section. The range of the hyperparameters are $\mathrm{n} \in\left\{n_{1}, n_{2}, \ldots, n_{l}\right\}$ and $\beta \in\left\{\beta_{1}, \beta_{2}, \ldots, \beta_{m}\right\}$. The hyperparameter search space then becomes the Cartesian product of the two sets. In the case of the grid search, each point of this search space is explored, which requires training the $\beta$-VAE and computing MIG. Grid search suffers from the curse of dimensionality since the number of evaluations exponentially grows with the size of the search space [31]. In comparison, the random search and BO algorithms do not search the entire space but search for a selected number of iterations $t$. While random search selects each point in the search space randomly, the BO algorithm performs a guided search using a Gaussian process model and the acquisition function.

In $\mathrm{BO}$ algorithm, the first $k$ iterations stabilize the Gaussian Process model using randomly selected points in the space to train a $\beta$-VAE and compute the MIG. After these iterations, the Gaussian process model is fitted to all the previously sampled hyperparameters. Then, the acquisition function based on expected improvement uses the posterior distribution of the Gaussian process model to find new hyperparameter(s) that may optimize the MIG. The new hyperparameter(s) are used to train a $\beta$-VAE and compute the MIG. So, this process is repeated for $t$ iterations, and in every iteration, a $\beta$-VAE is trained, and the MIG is computed as shown in Algorithm 2. This intelligent search mechanism based on prior information makes the search technique efficient as it takes a smaller number of points to explore in the search space as compared to both grid search and random search [31,65]. The $\mathrm{BO}$ algorithm has a polynomial time complexity because the most time consuming operation is the Gaussian process which takes polynomial time [31]. We report the experimental results for the three hyperparameter algorithms in Table 1. As seen in the Table, the $\mathrm{BO}$ algorithm takes the least time and iterations to select the hyperparameters that achieve the best MIG value as compared to the other algorithms.

\subsection{Latent Variable Mapping}

Given the data partition set $\mathcal{P}$ and a trained $\beta$-VAE that can generate the latent variable set $\mathcal{L}$, we find the most informative latent variables set $\mathcal{L}_{d} \subseteq \mathcal{L}$ that encodes information about the image features in the training set $\mathcal{T}$. As discussed earlier, the most informative latent variables form a separate cluster from the less informative ones when we partially disentangle the latent space using the $\mathrm{BO}$ heuristic. Although the most informative latent variables are separately clustered in the latent space, we need a mechanism to identify them. To do this, we present a KL-divergence based heuristic that is illustrated in Figure 5. 


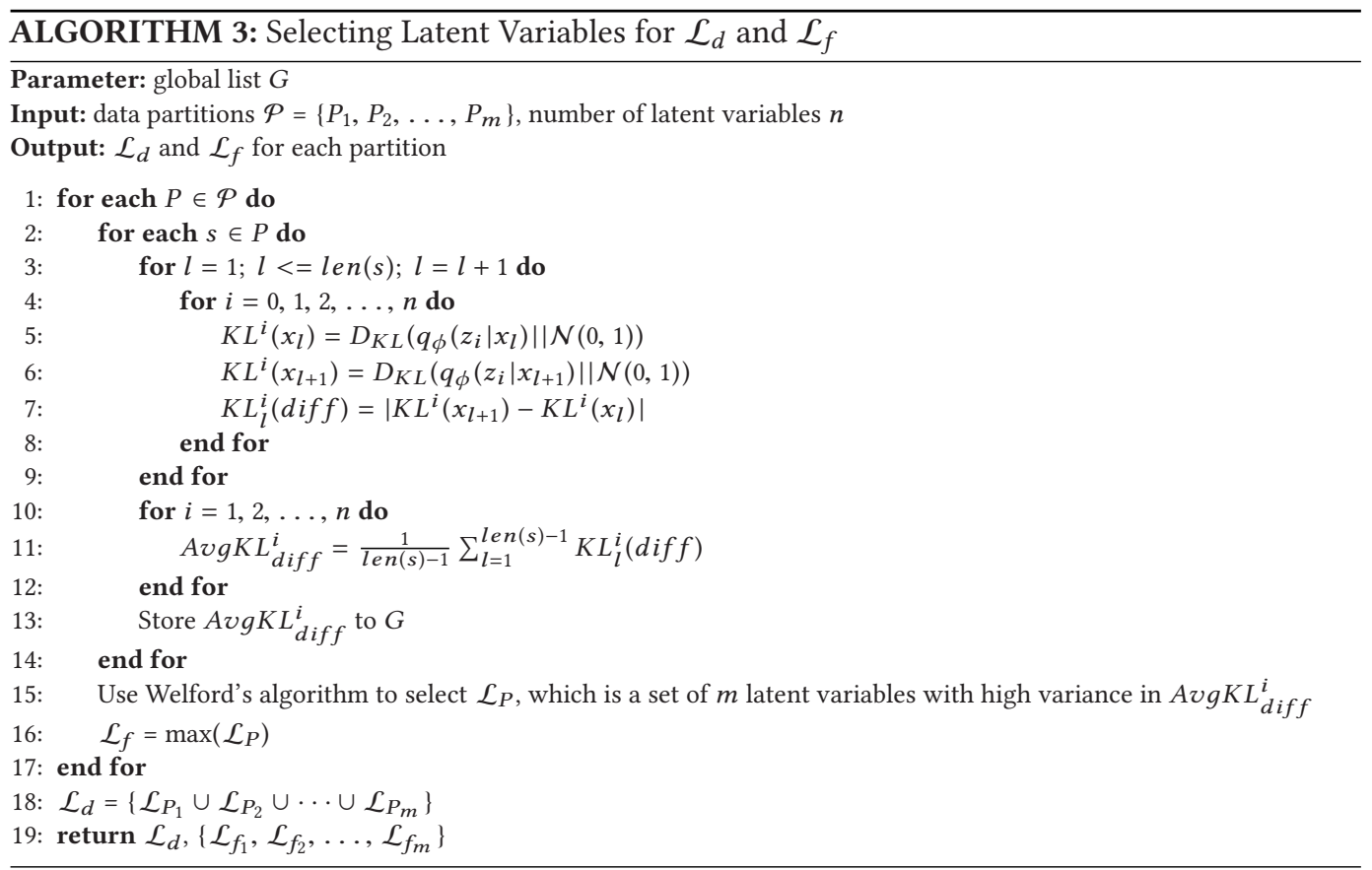

The latent variables in $\mathcal{L}_{d}$ are used as detectors i.e., they can detect overall distribution shifts in the images (we discuss the exact procedure later). However, this does not solve the problem of identifying the specific feature(s) whose labels caused the OOD. For this, given the representative feature $f$ of a partition has high variance, we identify the subset of latent variable(s) $\mathcal{L}_{f} \subseteq \mathcal{L}_{d}$ that is sensitive to the variations in $f$. For example, if we have a partition with images that have different values in the precipitation (e.g., precipitation $=20 \%$, precipitation $=50 \%$ ), then we map latent variable(s) that are sensitive to changes in precipitation level. The latent variable(s) in $\mathcal{L}_{f}$ is called the reasoner for feature $f$ and is used to identify if $f$ is responsible for the OOD. The steps in our heuristic are listed in Algorithm 3 and it works as follows: For each partition, $P \in \mathcal{P}$, and for each scene $s \in P$, we perform the following steps.

$\underline{\text { First, }}$, we take two subsequent images $x_{l}$ and $x_{l+1}$ and pass each of them separately to the trained $\beta$-VAE to generate the latent variable set $\mathcal{L}$ for each of the images. As a remainder, $\mathcal{L}$ is a collection of $n$ latent variables, and each latent variable has a latent distribution $(z)$ with parameters $\mu$ and $\sigma$. Then, for each latent variable of the images $(x)$ we compute a KL-divergence between its latent distribution $q\left(z_{i} \mid x\right)$ and the standard normal distribution $\mathcal{N}(0,1)$ as discussed in Section 3.1. The computed KL-divergence is $K L^{i}(x)=D_{K L}\left(q\left(z_{i} \mid x\right) \| \mathcal{N}(0,1)\right)$.

$\underline{\text { Second, }}$, we calculate the KL-divergence difference between corresponding latent variables of the two images as: $K L_{l}^{i}(\operatorname{diff})=\left|K L^{i}\left(x_{l+1}\right)-K L^{i}\left(x_{l}\right)\right|$. This procedure is repeated across all the subsequent images in the scene $s$.

Third, we compute an average KL-divergence difference for each latent variable across all the subsequent images of the scene as follows.

$$
\operatorname{AvgKL} L_{\text {diff }}^{i}=\frac{1}{\operatorname{len}(s)-1} \sum_{l=1}^{\operatorname{len}(s)-1} K L_{l}^{i}(\operatorname{diff}),
$$

where $K L_{l}^{i}(\operatorname{diff})$ is the KL-divergence difference of the latent variable $L_{i}$ for the $l^{\text {th }}$ subsequent image pair in a scene $s$ and $\operatorname{len}(s)$ is the number of images in $s$. This value indicates the average 


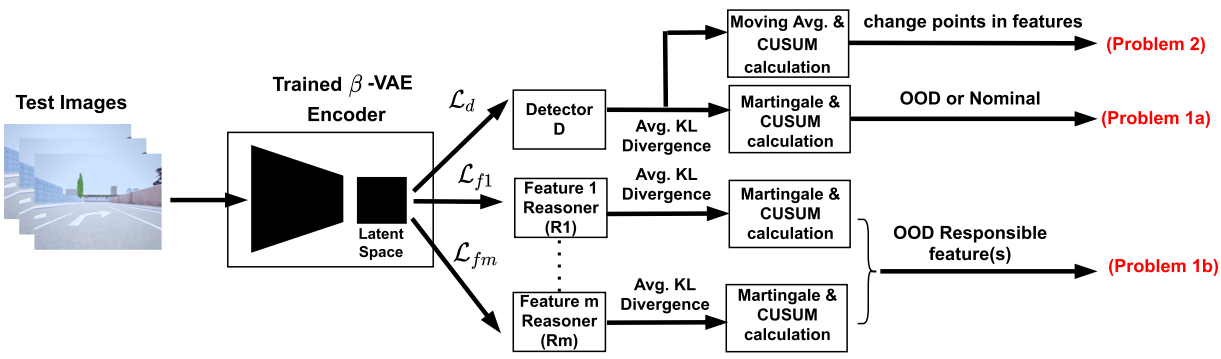

Fig. 6. Runtime OOD detection pipeline. The trained $\beta$-VAE detector at runtime provides three outputs that are sent to the decision manager to select an appropriate controller or control action that can mitigate the OOD problems as discussed in Section 2.

variance in the KL-divergence value across each latent variable for all the images in the $s$. This approach of computing the variations across $s$ is motivated from the manual latent variable mapping technique in [29]. Further, the $A v g K L_{\text {diff }}^{i}$ value is computed $\forall s \in P$.

Fourth, we then use the Welford's variance calculator [79] to compute the variance in the $A v g K L_{\text {diff }}^{i}$ value across all the scenes in the partition. Welford's variance calculator computes and updates the variance in a single pass as the measurements are available. It does not require storing the measurements till the end for the variance calculation, which will make the variance calculation across several scenes faster. In our case, the variance calculator returns a partition latent variable set $\mathcal{L}_{P}$, which is a set of top $m$ latent variables that has the highest $A v g K L_{\text {diff }}^{i}$ across all images in $P$. Selecting an appropriate number of latent variables $(m)$ for $\mathcal{L}_{P}$ is crucial, as we use it to select the latent variables for $\mathcal{L}_{d}$ and $\mathcal{L}_{f}$. The value for $m$ is chosen empirically, and the selection depends on the variances across the scenes of a partition. However, it is important to note that selecting a small value for $m$ may not include all the informative latent variables required for detection and choosing a large value for $m$ may include uninformative latent variables that may reduce the detection accuracy and sensitivity.

Further, we choose the top latent variable(s) from $\mathcal{L}_{P}$ and use it as the reasoner $\left(\mathcal{L}_{f}\right)$ for the partition. If the partition has variance in an independent image feature (e.g., brightness), then a single best latent variable which has the most sensitivity can be used as the reasoner. However, if the feature is not independent, then more than one latent variable needs to be used, and the size of $\mathcal{L}_{P}$ increases. Besides, if two features correlate, then a single latent variable may be sensitive to both features. If such a latent variable is used for reasoning at runtime, and if it shows variation, then we attribute both the features to be responsible for the OOD.

Finally, these steps are repeated for all the partitions in $\mathcal{P}$, and the latent variables for $\mathcal{L}_{d}$ is formed by $\left\{\mathcal{L}_{P_{1}} \cup \mathcal{L}_{P_{2}} \cup \cdots \cup \mathcal{L}_{P_{m}}\right\}$. If the latent space is partially disentangled, then the top $m$ latent variables in each $\mathcal{L}_{P}$ will mostly be the same. Otherwise, the number of similar latent variables in each $\mathcal{L}_{P}$ will be small.

\subsection{Runtime Out-of-Distribution Detection}

At runtime, we use the trained $\beta$-VAE and the latent variable set $\mathcal{L}_{d}$ and $\mathcal{L}_{f}$ to detect OOD problems discussed in Section 2. Figure 6 shows the pipeline for runtime OOD detection, and it works as follows. As a test image $x_{t}$ is observed, the encoder of the trained $\beta$-VAE is used to generate the latent space encoding. Then, the respective latent variables in $\left(\mathcal{L}_{d}, \mathcal{L}_{f}\right)$ are sent to different processes to compute the average KL-divergence between the latent variables in $\mathcal{L}_{d}$ or latent variable(s) in $\mathcal{L}_{f}$ for the identified features. The KL-divergence is computed between each latent variables in 


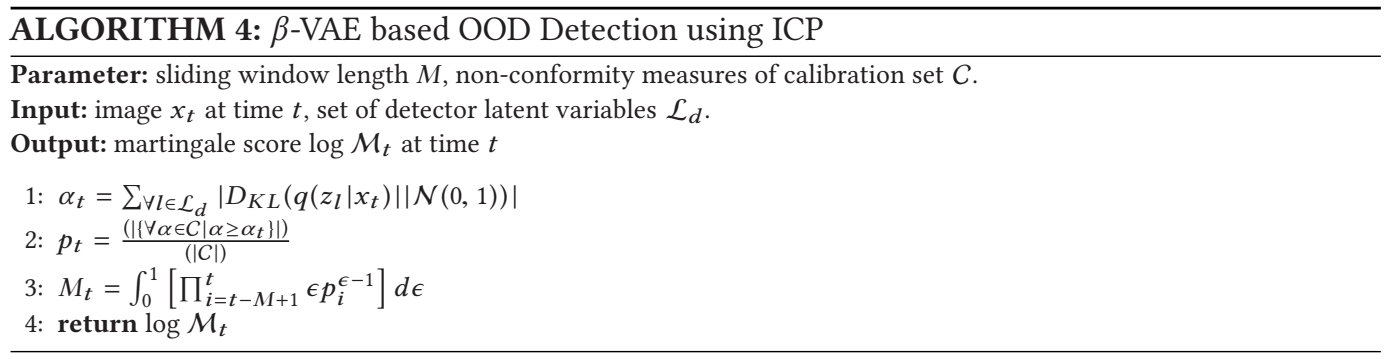

$\mathcal{L}_{d}$ and $\mathcal{L}_{f}$, and the normal distribution $\mathcal{N}(0,1)$ as shown in Equation (6).

$$
\alpha_{t}=K L\left(x_{t}, \mathcal{N}(0,1)\right)=\frac{1}{L} \sum_{l=1}^{L}\left|D_{K L}\left(q\left(z_{l} \mid x_{t}\right)|| \mathcal{N}(0,1)\right)\right|,
$$

where $L$ is the number of selected latent distributions; for the detector, it is the number of latent variables in $\mathcal{L}_{d}$, and for each feature, it is the number of latent variables in $\mathcal{L}_{f}$.

To detect the first OOD problem (Problem 1), the KL-divergence is used as the non-conformity score to compute the ICP and martingale score as shown in Algorithm 4. However, as the martingale can grow large very rapidly, we use the log of martingale. Then, a CUSUM over the log of martingale is computed to identify when the martingale goes consistently high. The CUSUM value $S_{t}$ of the detector latent variables $\mathcal{L}_{d}$ is compared against a detector threshold $\left(\tau_{d}\right)$ to detect if the image $x_{t}$ is OOD compared to the calibration set. Then, the CUSUM value $S_{t}$ of the reasoner latent variables $\mathcal{L}_{f}$ is compared against a reasoner threshold $\left(\tau_{r}\right)$ to identify if the known feature(s) is responsible for the OOD as discussed in Problem $1 b$ of Section 2. The thresholds are empirically tuned as a tradeoff between false positives and mean detection delay [3].

To detect the second OOD problem (Problem 2), the latent variables in $\mathcal{L}_{d}$ is used with sliding window moving average and CUSUM for change point detection. For this, the average KLdivergence of all the latent variables in $\mathcal{L}_{d}$ is computed using Equation (6), and a moving average of the average KL-divergence $\left(A_{K L}\right)$ is computed over a sliding window $\left[x_{t-M+1}, \ldots, x_{t}\right]$ of previous $M$ images in the time series. $A_{K L}$ is used to compute the CUSUM value $S_{t}$, which is compared against a threshold $\tau_{c p}$ to detect changes.

Finally, the outputs of the detector (See Figure 6) are sent to the decision manager, which can use these detection results to perform system risk estimation [26] or high level controller selection using simplex strategies [64]. In this work, we use a simple decision logic that uses the OOD detection result to perform a control arbitration from the DNN controller to an autopilot controller.

\section{EXPERIMENTS AND RESULTS}

We evaluate our approach using an AV example in the CARLA simulator [14] and show the preliminary results from the real-world nuImages dataset [52]. The experiments ${ }^{2}$ in this section were performed on a desktop with AMD Ryzen Threadripper 16-Core Processor, 4 NVIDIA Titan Xp GPU's, and $128 \mathrm{GiB}$ memory.

\subsection{System Overview}

Our first example system is an AV which must navigate different road segments in town 1 of the CARLA simulator. The architecture of our AV, shown in Figure 7, relies on a forward-looking camera for perception and a speedometer for measuring the system's speed. It uses the NVIDIA

\footnotetext{
${ }^{2}$ source code to replicate these experiments can be found at https://github.com/scope-lab-vu/Beta-VAE-OOD-Detector.
} 


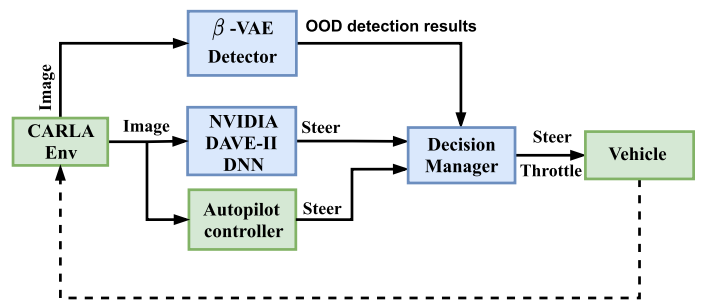

Fig. 7. AV block diagram. The components in green come inbuilt with the CARLA simulator, while the components in blue are designed for our example. While the simulator requires a GPU, the other components are run on one $\mathrm{CPU}$ core to emulate a resource constrained setting.

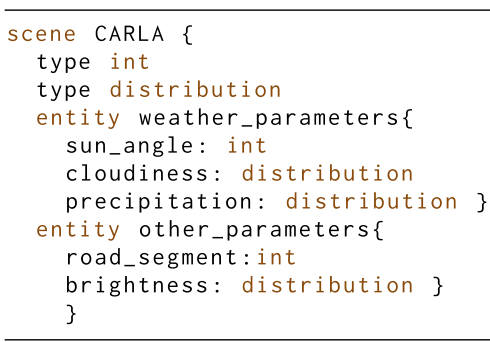

\begin{tabular}{|c|c|c|c|c|c|c|}
\hline Feature & $\begin{array}{c}\text { Training } \\
\text { Range }\end{array}$ & Nom & HP & HB & HPB & NR \\
\hline Sun Angle & 90 & 90 & 90 & 90 & 90 & 90 \\
\hline Cloudiness & {$[0,50]$} & 25 & 50 & 50 & 25 & 50 \\
\hline Precipitation & {$[0,50]$} & 0 & $\mathbf{4 0}$ & $\mathbf{0}$ & 50 & $\mathbf{0}$ \\
\cline { 4 - 7 } & & & 60 & 0 & 75 & 0 \\
\hline Brightness & {$[0,50]$} & 0 & $\mathbf{0}$ & 25 & 40 & $\mathbf{0}$ \\
\cline { 4 - 8 } & $1,2,3$ & 3 & 2 & 2 & 2 & 7 \\
\hline Road & & & & & \\
\hline
\end{tabular}

Fig. 8. Data generation. (left) A fragment of our Scenario Description Language. (right) Scenes in CARLA simulation: Nominal scene $(\mathrm{Nom})$ is generated by randomly sampling the features in their training ranges. High Precipitation $(H P)$, High Brightness $(H B)$, High Precipitation and Brightness $(H P B)$ scene, and New Road $(N R)$ are test scenes for which the feature values change from a training range value to a value outside the range at $t=20$ seconds. The initial values of precipitation and brightness are highlighted in green.

DAVE-II DNN [6] as the primary controller and the simulator's inbuilt autopilot mode as the secondary safety controller. In addition, a trained $\beta$-VAE detector and reasoner are used in parallel to the two controllers. The detection results and the steering values from the two controllers are sent to a simplex decision manager, which selects the appropriate steering value for the system based on the detection result. That is, if the detector returns the image to be OOD, the decision manager selects the autopilot controller to drive the AV. The sampling period used in the simulation is $1 / 13$ seconds, and the vehicle moves at a constant speed of $0.5 \mathrm{~m} / \mathrm{s}$ for all our experiments.

5.1.1 Operating Modes. Our AV has two operating modes: (a) manual driving mode, which uses CARLA's autopilot controller to drive around town 1 . The autopilot controller is not an AI component, but it uses hard-coded information from the simulator for safe navigation. We use this mode to collect the training set $\mathcal{T}$ and the test set $\left(\mathcal{T}_{t}\right)$. These datasets are a collection of several CARLA scenes generated by a custom Scenario Description Language (SDL) shown in Figure 8; and (b) autonomous mode, which uses a trained NVIDIA DAVE-II DNN controller to drive the AV. In this setup, the $\beta$-VAE detector is used in parallel to the DNN controller to perform OOD detection.

5.1.2 Data Generation. Domain-specific SDLs such as Scenic [20] and MSDL [19] are available for probabilistic scene generation. However, they did not fit our need to generate partition variations. Hence, we have implemented a simple SDL in the textX [11] meta language (See Figure 8), which is combined with a random sampler over the range of the simulator's features like sun altitude, cloudiness, precipitation, brightness, and road segments to generate different scenes. 

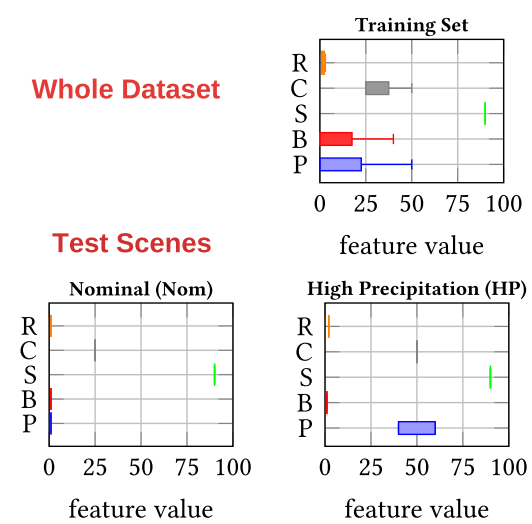

feature value

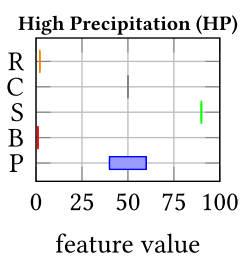

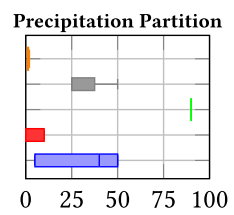

feature value
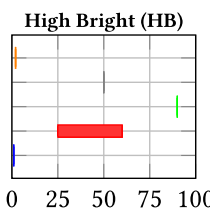

feature value

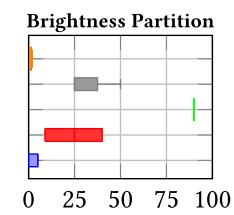

feature value

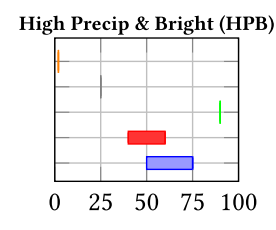

feature value

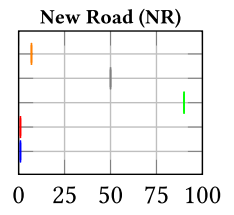

feature value

$\begin{array}{llll}\text { Precipitation } & \text { Brightness } & \text { Sun Angle } & \text { Cloudiness } \\ \text { Road Segment }\end{array}$

Fig. 9. Feature value variance. Plots representing the normalized values of sun angle (S), cloudiness (C), precipitation $(\mathrm{P})$, brightness $(\mathrm{B})$, and road segments $(\mathrm{R})$ for the training set, partitions, and the test scenes. We use the test scene abbreviations Nom (Nominal), HP (High Precipitation), HB (High Brightness), HPB (High Precipitation and Brightness), and NR (New Road segment) for the rest of the article. The NR scene has all the feature values like the Nom scene, but it uses the road segment 7 that is not in the training distribution.

Train Scenes: The training feature labels, and their values are shown in Figure 8. These features were randomly sampled in the ranges shown in Figure 8 to generate eight scenes of 750 images each that constituted the training set $\mathcal{T}$. Among these, two scenes had precipitation of $0 \%$, the brightness of $0 \%$, sun angle $90^{\circ}$, cloudiness of $25 \%$, and road segment of 1 and 2 for each scene, respectively. Three scenes had different precipitation values (precip $=5 \%$, precip $=40 \%$, precip $=50 \%$ ) while sun angle took a value of $90^{\circ}$, cloudiness took a value of $25 \%$, brightness took a value of $0 \%$, and $10 \%$, and the road segments took a value of 1 and 2 . The remaining three scenes had different brightness values (bright $=9 \%$, bright $=25 \%$, bright $=40 \%$ ), precipitation took a value of $0 \%$ and $5 \%$, and all the other parameters remained the same as the other scenes. We split 6,000 images of $\mathcal{T}$ into 4,000 images of $\mathcal{T}_{P}$ and 2,000 images of $C$ in the standard 2:1 ratio (page 222 of [27]) for ICP calculations.

Test Scenes: The test scenes included a nominal scene (Nom) and four OOD scenes as shown in Figure 8. Each scene was 20 seconds long, and it had 260 images. (1) Nominal Scene (Nom) was an in-distribution scene generated from the training distribution. (2) High Precipitation $(H P)$ scene was an OOD scene in which the precipitation was increased from $40 \%$ (in training range) to $60 \%$ (out of training range) at $t=2$ seconds. (3) High Brightness $(H B)$ scene was an OOD scene in which the brightness was increased from 25\% (in training range) to $60 \%$ (out of training range) at $t=2 \mathrm{sec}-$ onds. (4) High Precipitation and Brightness $(H P B)$ scene was also an OOD scene in which both the precipitation and the brightness were increased out of the training range at $t=2$ seconds. (5) New Road $(N R)$ scene was an OOD scene with a new road segment (segment $=7$ ) that was not in the training range, but the other features remained within the training range. Figure 9 shows the variance in the normalized values of the scene features for the training set, partitions, and test scenes.

\section{$5.2 \beta$-VAE Detector}

We implement the steps of our approach discussed in Section 4 to design a $\beta$-VAE detector for our AV example.

5.2.1 Data Partitioning. Using our partitioning technique discussed in Section 4.1, we select three scenes with variance in precipitation values (precip $=5 \%$, precip $=40 \%$, precip $=50 \%$ ) as 
Table 1. Comparing Hyperparameter Search Algorithms

\begin{tabular}{|c|c|c|c|c|c|}
\hline Algorithm & \# of Iterations & $\begin{array}{c}\text { Iterations to } \\
\text { reach optimum }\end{array}$ & $\begin{array}{c}\text { Search Time } \\
\text { (min) }\end{array}$ & max MIG & Selected Parameter \\
\hline Grid & 360 & 5 & 10924.55 & 0.0017 & $30,1.4$ \\
\hline Random & 50 & 40 & 1199.51 & 0.00032 & $40,1.5$ \\
\hline BO & $\mathbf{5 0}$ & $\mathbf{1 6}$ & $\mathbf{8 3 7 . 0 5}$ & $\mathbf{0 . 0 0 1 8}$ & $30, \mathbf{1 . 4}$ \\
\hline
\end{tabular}

Bayesian Optimization Algorithm is Compared Against Random and Grid Search Algorithms.

the precipitation partition $P 1$, and the remaining three scenes with variance in brightness values (bright $=9 \%$, bright $=25 \%$, bright $=40 \%$ ) as the brightness partition $P 2$.

5.2.2 Latent Space Encoding. We applied the BO algorithm based heuristic discussed in Section 4.2 to select and train the $\beta$-VAE with appropriate hyperparameters.

Network Structure and Training: We designed a $\beta$-VAE network that has four convolutional layers $32 / 64 / 128 / 256$ with $5 \times 5$ filters and $2 \times 2$ max pooling followed by four fully connected layers with $2,048,1,000,250$, and 50 neurons. A symmetric deconvolutional decoder structure is used as a decoder. This network along with images in $\mathcal{T}$ was used in the $\mathrm{BO}$ algorithm discussed in Algorithm 2. For each iteration of the $\mathrm{BO}$ algorithm, the network was trained for 100 epochs using the Adam gradient-descent optimizer and a two-learning scheduler, that had an initial learning rate $\eta=1 \times 10^{-5}$ for 75 epochs, and subsequently fine-tuning $\eta=1 \times 10^{-6}$ for 25 epochs. Learning rate scheduler is used to improve the model's accuracy and explore areas of lower loss. In addition, we had an early stopping mechanism to prevent the model from overfitting.

In addition to network training, the algorithm also involved computing the MIG in every iteration. For computing it, we utilized the images and labels from partitions $P 1$ and $P 2$, which were generated in the previous step. To obtain robust MIG, we computed the latent variable entropy by randomly sampling 500 samples from each latent variable in the latent space. To back this, we also averaged the MIG across five iterations.

Performance Comparison: We compared the performance of $\mathrm{BO}$, grid, and random search algorithms. The results of these algorithms are shown in Table 1. Random search and BO algorithm was run for 50 trials, while the grid search was run for 360 trials across all combinations of $\mathrm{n} \in$ $[30,200]$ and $\beta \in[1,5]$. In comparison, the BO algorithm achieved the highest MIG value of 0.0018 for $\beta=1.4$ and $n=30$ hyperparameters. It also took the shortest time of 837.05 minutes (early termination because optimal hyperparameters(s) were found) as compared to the other algorithms.

5.2.3 Latent Variable Mapping. We used the selected and trained $\beta$-VAE along with the data partitions $P 1$ and $P 2$ to find latent variables for OOD detection $\left(\mathcal{L}_{d}\right)$ and reasoning $\left(\mathcal{L}_{f}\right)$. For each scene in the partitions, we applied the steps in Algorithm 3 as follows. First, we used the successive images in each scene to generate a latent variable set $\mathcal{L}$ and then computed a KL-divergence value.

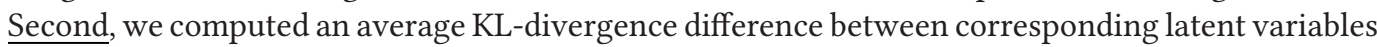
of the two images. Third, we computed the average KL-divergence difference (using Equation (5)) for each latent variable across all the subsequent images in a scene. We repeated these steps for all the scenes in both partitions. Finally, for each partition we identified a partition latent variable set $\mathcal{L}_{P}$ using the Welford's algorithm as discussed in Section 4.3. The number of latent variables $m$ in $\mathcal{L}_{P}$ requires selection based on the dataset. In this work, the value for $m$ is chosen by human judgment. For the CARLA dataset, we chose the value of $m$ to be 4 .

Implementing these steps, we selected the partition latent variable sets $\mathcal{L}_{P 1}=\left\{L_{2}, L_{25}, L_{20}\right.$, $\left.L_{0}, L_{29}\right\}$ for $P 1$ and $\mathcal{L}_{P 2}=\left\{L_{25}, L_{0}, L_{20}, L_{2}, L_{29}\right\}$ for $P 2$. These latent variables had the highest KL-divergence variance values and hence were selected. The KL-divergence variance values of these latent variables are reported in Table 2. Then, the union of these two partition sets were 
Table 2. Latent Variable Mapping: Ordered List of Latent Variable Set

$\mathcal{L}_{P}$ for $P 1$ and $P 2$

\begin{tabular}{|c|c|c|c|c|}
\hline Partition & \multicolumn{4}{|c|}{ Latent Variable set $\mathcal{L}_{P}$} \\
\hline P1 & $L_{2}(\mathbf{0 . 0 9})$ & $L_{25}(0.06)$ & $L_{0}(0.05)$ & $L_{29}(0.02)$ \\
\hline P2 & $L_{25}(\mathbf{0 . 1 6})$ & $L_{0}(0.09)$ & $L_{20}(0.07)$ & $L_{2}(0.07)$ \\
\hline
\end{tabular}

The latent variable $L_{2}$ had highest KL-divergence variance across the scenes in $P 1$. $L_{25}$ had the highest KL-divergence variance across the scenes in $P 2 . \mathcal{L}_{d}$ is chosen as the union of the two $\mathcal{L}_{P}$ sets. Chosen $\mathcal{L}_{d}=\left\{L_{0}, L_{2}, L_{20}, L_{25}, L_{29}\right\}$.

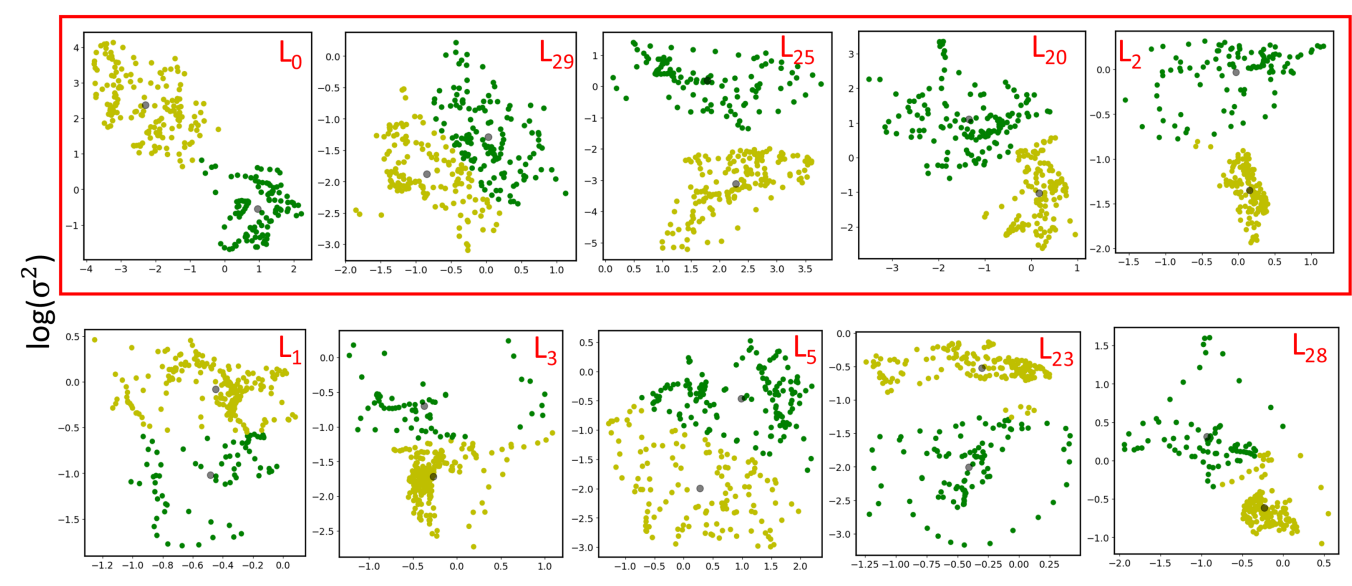

Fig. 10. Scatter plots of individual latent variables. The latent distributions of the selected latent variables (highlighted in red) as compared to 5 other latent variables that were not selected. Yellow points represent the distributions of the train images, and the green points represent the distributions of the test images that were OOD. The selected latent variables into a well-formed cluster, and there is a higher separation between the train and the test image clusters. The un-selected latent variables do not form clean clusters. Using the 5 most informative latent variables for detection resulted in better robustness and minimum sensitivity as compared to using all the latent variables as shown in Table 3. Plot axis: $x$-axis represents the mean of the latent distributions in the range $[-5,5]$, and the $y$-axis represents the log of variance in the range $[-5,5]$.

used as the total detector $\mathcal{L}_{d}=\left\{L_{0}, L_{2}, L_{20}, L_{25}, L_{29}\right\}$. Figure 10 shows the scatter plots of the latent distributions of the selected latent variables and 5 randomly selected latent variables $\left(L_{1}, L_{3}, L_{5}\right.$, $\left.L_{15}, L_{28}\right)$ for the train images (yellow points) and the test images (green points), which are OOD with high brightness. The latent distributions of the selected latent variables highlighted in the red box form evident clusters between the train images and the test images, and these clusters have a good intra-cluster separation. But, for the other latent variables, the distributions are scattered and do not form clean clusters, and these clusters are not well separated.

Further, we chose one latent variable with the maximum variance in the partition latent variable sets and, it was used as the reasoner for that partition. We chose $L_{2}$ as the reasoner for the precipitation partition $P 1$ and $L_{25}$ is used as the reasoner for the brightness $P 2$. Our decision of choosing only one latent variable for reasoning is backed by the fact that the dataset was synthetically generated, and the features were not highly correlated. However, real-world datasets may require more than one latent variable for reasoning.

\subsection{Out-of-Distribution Detection Results from CARLA Simulation}

5.3.1 Evaluation Metrics. (1) Precision $(P)$ is a fraction of the detector identified anomalies that are real anomalies. It is defined in terms of true positives (TP), false positives (FP), and false 

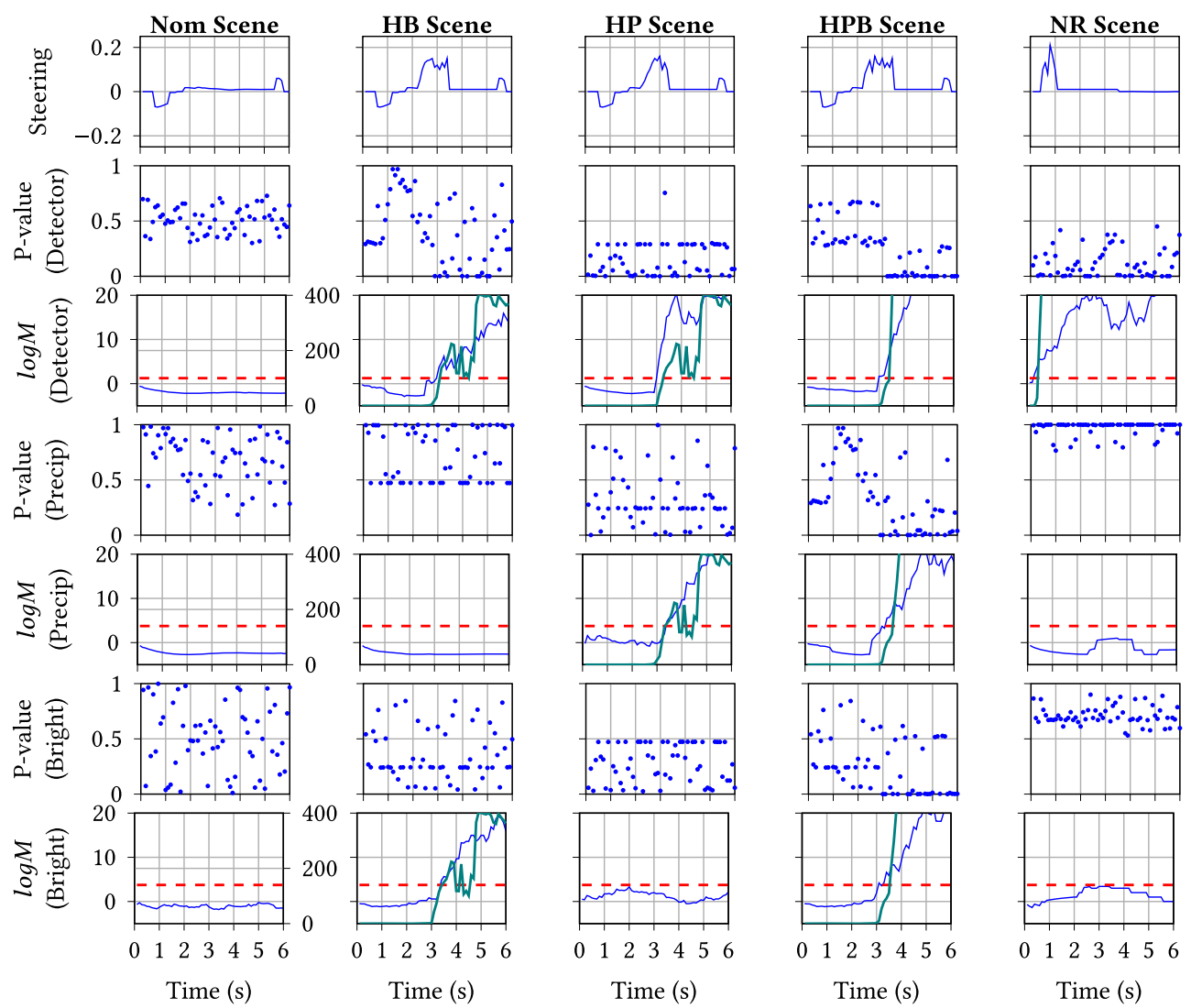

$\begin{array}{lllllll}0 & 1 & 2 & 3 & 4 & 5 & 6\end{array}$

Time (s)

Fig. 11. Runtime OOD detection. Performance of the $\beta$-VAE detector for the 5 test scenes generated in Section 5.1. The solid blue line represents the log of martingale, the solid green lines represent the CUSUM values, and the dotted red lines represent the threshold $(\tau)$ for CUSUM comparison. Further, the left $y$-axis shows the log of martingale with range $[-5,20]$, and the right $y$-axis shows the CUSUM value with range $[0,400]$. The cusum threshold represented in the red dotted lines are plotted to the right $y$-axis.

negatives $(\mathrm{FN})$ as $T P \div(T P+F N)$. (2) Recall $(R)$ is a fraction of all real anomalies that were identified by the detector. It is calculated as $T P \div(T P+F P)$. The FP and FN for the test scenes is shown in Table 4. (3) F1-score is a measure of the detector's accuracy that is computed as $2 \times(P \times R) \div(P+R)$. (4) Execution Time is computed as the time the detector receives an image to the time it computes the log of martingale. (5) Average latency is the number of frames between the detection and the occurrence of the anomaly. (6) Memory Usage is the memory utilized by the detector.

5.3.2 Runtime OOD Detection. We evaluate the performance of the selected $\beta$-VAE network ( $\beta=1.4$ and $n=30$ ) for the 5 test scenes described in Figure 8. Additional hyperparameters that were used by the detectors and the reasoners are as follows. The martingale sliding window size $M=20$, the CUSUM parameters for the detector are $\omega_{d}=14$ and $\tau_{d}=100$, and for the reasoners are $\omega_{r}=18$ and $\tau_{r}=130$. These hyperparameters were selected empirically based on the false positive results from several trial runs. Figure 11 summarizes the detector's performance for a short segment of the 5 test scenes. For the $H P, H B, H P B$, and $N R$ scenes, the scene shifts from in-distribution to OOD at $t=2$ seconds, and they are used to illustrate the detection and reasoning capability of our approach. 

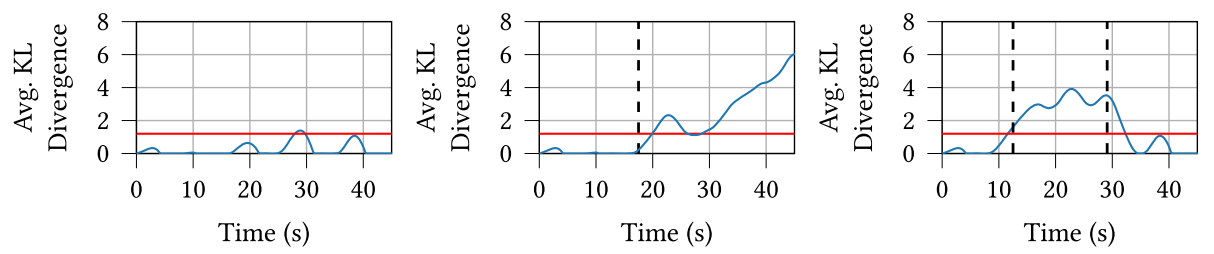

- CUSUM Threshold - - - Change Point

Fig. 12. Runtime change detection. Moving Average and CUSUM for identifying feature changes in Nom, $H B$, and an extended $H B$ test scenes. (Left) Nom scene - there is no change in the scene, so the average $\mathrm{KL}$-divergence remains below $\tau$. (Center) $H B$ scene - the brightness of the scene changes at 17.5 seconds and this was identified by the detector. (Right) Extended $H B$ scene - high brightness is introduced for a short period between 9.5 seconds to 29.5 seconds, and the detector was able to identify both the changes.

The Nom scene has all the feature values within the training distribution, and it is used to illustrate the detector's ability to identify in-distribution images. As seen in Figure 11, the martingale of the detector and both the reasoners remain low throughout. In $H P$ and $H B$ scenes, the precipitation and the brightness feature values increase out of the training distribution at $t=2$ seconds. In the HP scene, the martingales of the detector and the reasoner for the precipitation feature increase above the threshold after $t=2$ seconds. However, as seen martingale of the reasoner for brightness does not increase. So, we conclude that the precipitation feature is the reason for the OOD. In the $H B$ scene, the martingales of the detector and the reasoner for the brightness feature increase above the threshold after $t=2$ seconds. Also, the martingale of the reasoner for precipitation shows a slight variation but does not increase above the threshold. So, we conclude the brightness feature is the cause of the OOD. In the HPB scene, the precipitation and brightness feature increase to a value out of the training distribution at $t=2$ seconds. The martingale of the detector and both the feature reasoners increase above the threshold. So, we attribute both the features to be the cause of the OOD. The peaks in the steering plots at $t=2$ seconds are when the DAVE-II DNN steering predictions get erroneous, and the decision manager arbitrates the control to the autopilot controller.

The NR scene is of interest for this work, as the scene has a new road segment with different background artifacts (e.g., buildings, traffic lights) that were not in the training distribution. But the precipitation and brightness feature values were within the training distribution. When tested on this scene, the detector martingale instantly increases above the threshold, identifying the scene to be OOD. However, the martingales of the reasoners for precipitation and brightness only show slight variations without increasing beyond the threshold. The result implies that the scene is OOD, but a feature other than brightness and precipitation has varied and is responsible for the OOD.

Further, Figure 12 shows the plots of the capability of the $\beta$-VAE detector in identifying changes in the current test image as compared to the previous images in the time series (problem2). For these evaluations, we used the Nom, $H B$, and extended $H B$ test scenes, which had a length of 50 seconds each. The Nom and $H B$ scenes are same as the previous setup, but in the extended $H B$ scene, the brightness feature value abruptly increases only for a brief period between 9.5 seconds and 29.5 seconds. For these test scenes, we performed the moving average calculation on a sliding window of $M=20$ with the CUSUM parameters of $\omega_{c p}=0.75$ and $\tau_{c p}=1.2$. With these parameters, our detector could identify change points with a short latency of 11 frames, which translates to one second of inference time for the AV system.

5.3.3 Evaluating the Design Approach. Table 3 illustrates how the proposed heuristics in each step of our approach (Section 4) results in achieving the best detector properties (text highlighted 
Table 3. Design Approach Evaluation

\begin{tabular}{|c|c|c|c|c|c|c|}
\hline \multicolumn{4}{|c|}{ Design-time steps of our approach } & \multicolumn{3}{|c|}{ Detector Properties } \\
\hline \multirow[b]{2}{*}{ Partitioning } & \multicolumn{2}{|c|}{ Latent Space Encoding } & \multirow{2}{*}{$\begin{array}{l}\text { Selected } \mathcal{L}_{d} \\
\text { for Detection }\end{array}$} & \multirow{2}{*}{$\begin{array}{l}\text { Robustness } \\
\text { F1-score (\%) }\end{array}$} & \multirow{2}{*}{$\begin{array}{c}\text { Minimum } \\
\text { Sensitivity (\%) }\end{array}$} & \multirow{2}{*}{$\begin{array}{l}\text { Execution } \\
\text { Time (ms) }\end{array}$} \\
\hline & $\begin{array}{l}\text { Objective } \\
\text { Function }\end{array}$ & $\begin{array}{c}\text { Optimization } \\
\text { Algorithm }\end{array}$ & & & & \\
\hline \multirow{6}{*}{ Yes } & \multirow{3}{*}{ MIG } & $\mathrm{BO}(30,1.4)$ & {$[0,2,20,25,29]$} & 96.98 & 96 & 74.09 \\
\hline & & Grid $(30,1.4)$ & {$[0,2,20,25,29]$} & 96.98 & 96 & 74.09 \\
\hline & & Random $(40,1.5)$ & {$[0,17,6,8,7]$} & 80.75 & 35 & 79.15 \\
\hline & \multirow{3}{*}{ ELBO } & $\mathrm{BO}(30,1.0)$ & {$[0,1,6,21,23]$} & 95.83 & 63 & 75.39 \\
\hline & & Grid $(40,1.0)$ & {$[13,28,26,23,0]$} & 84.65 & 54 & 78.85 \\
\hline & & Random $(30,1.2)$ & {$[10,14,21,22,26]$} & 94.9 & 71 & 74.98 \\
\hline \multirow{3}{*}{ No } & \multirow{3}{*}{ ELBO } & $\mathrm{BO}(30,1.0)$ & All 30 & 85.89 & 73 & 379.47 \\
\hline & & Grid $(40,1.0)$ & All 40 & 68.96 & 42 & 488.85 \\
\hline & & Random $(30,1.2)$ & All 30 & 89.73 & 53 & 396.89 \\
\hline
\end{tabular}

Evaluations of How the Heuristics of Our Design Approach Influence the Detector Properties Discussed in Section 2. We evaluated Robustness on the Nom, HPB, and NR scenes. Minimum sensitivity was evaluated on the HP and $H B$ scenes. Resource efficiency was measured across all the test scenes. The numbers in the optimization algorithm column indicate the selected hyperparameters. Text in green highlights the best detector properties achieved by our approach. Text in red highlights a high detection time.

in green). The properties of interest are robustness, minimum sensitivity, and resource efficiency, which are defined in Section 2. We evaluate robustness on the in-distribution scene Nom, and two OOD scenes $H P B$ and $N R$. The $H P$ and $H B$ scenes had variations in either the precipitation value or the brightness value, but they did not have simultaneous variations in both. So, it was suitable to use them to measure the detector's minimum sensitivity towards these features. However, in the $H P B$ scene, both these features varied simultaneously, so it was not suitable for measuring the minimum sensitivity. The resource efficiency was measured across all the scenes. For these evaluations, we have compared the proposed heuristics to an alternate technique in each step. The comparisons are as follows (proposed techniques are underlined): (1) MIG vs. ELBO loss function (discussed in Section 3), (2) Bayesian Optimization vs. Grid and Random Search, and (3) Selective vs. All latent variables for detection. Our evaluations are as follows.

If the dataset can be partitioned, either MIG or ELBO can be used as the objective function with the $\mathrm{BO}$, grid, or random search algorithms. Since the dataset can be partitioned, the latent variable heuristic could be applied to select a subset of latent variables, which can be used for detection. As illustrated in Table 3, the optimization algorithm and objective function combinations resulted in 5 different $\beta$-VAE networks and 5 latent variables for $\mathcal{L}_{d}$. Among these, the $\mathrm{BO}$ and grid algorithms using MIG resulted in the best detector that had robustness of $96.98 \%$, minimum sensitivity of $96 \%$, and a detection time of 74.09 milliseconds. In comparison, the other detectors had low robustness and minimum sensitivity, and a similar detection time.

However, if the dataset cannot be partitioned, then ELBO is the only objective function that can be used with the $\mathrm{BO}$, grid, or random search algorithms. However, without partitioning, the latent variable mapping heuristic cannot be applied. So, all the latent variables for the chosen $\beta$-VAE had to be used for detection. Using all the latent variables for detection resulted in a less robust and sensitive detector that took an average of 400 milliseconds (text in red) as shown in Table 3.

\subsection{Detection Results from Competing Baselines}

We compare the performance of the $\beta$-VAE detector to other state-of-the-art approaches using our AV example in CARLA. The approaches that we compare against are: (1) Deep-SVDD one-class classifier; (2) VAE based reconstruction classifier; (3) chain of one-class Deep-SVDD classifiers; and (4) chain of VAE based reconstruction classifiers. The VAE network architecture is the same as that of $\beta$-VAE (described in Section 5) but uses the hyperparameters of $\beta=1$ and $n=1,024$. The 
Table 4. Metrics: Definitions of False Positives and False Negatives for the $H P, H B, H P B$, and $N R$ Test Scenes

\begin{tabular}{|c|l|l|}
\hline Scene & \multicolumn{1}{|c|}{ FP } & \multicolumn{1}{|c|}{ FN } \\
\hline$H P$ & $\begin{array}{l}\text { The image is not OOD due to high precipitation, } \\
\text { but it is identified as OOD. }\end{array}$ & $\begin{array}{l}\text { The image is OOD due to high rain, but is } \\
\text { identified as in-distribution }\end{array}$ \\
\hline$H B$ & $\begin{array}{l}\text { The image is not OOD due to high } \\
\text { brightness, but is identified as OOD. }\end{array}$ & $\begin{array}{l}\text { The image is OOD due to high brightness, } \\
\text { but is identified as in-distribution. }\end{array}$ \\
\hline$H P B$ & $\begin{array}{l}\text { The image is not OOD due to high precipitation } \\
\text { and high brightness but, is identified as OOD. }\end{array}$ & $\begin{array}{l}\text { The image is OOD due to high rain and high } \\
\text { brightness, but is identified as in-distribution. }\end{array}$ \\
\hline$N R$ & $\begin{array}{l}\text { The image is not OOD due to change in road } \\
\text { segment, but is identified as OOD. }\end{array}$ & $\begin{array}{l}\text { The image is OOD due to change in road } \\
\text { segment, but is identified as in-distribution. }\end{array}$ \\
\hline
\end{tabular}

one-class Deep-SVDD network has four convolutional layers of 32/64/128/256 with $5 \times 5$ filters with LeakyReLu activation functions and $2 \times 2$ max-pooling, and one fully connected layer with 1,568 units. These networks are also trained using a two-learning scheduler, with 100 epochs at a learning rate $\eta=1 \times 10^{-4}$, and 50 epochs at a learning rate $\eta=1 \times 10^{-5}$. Further, we combined two of these classifiers to form a chain of Deep-SVDD classifiers and a chain of VAE classifiers. In each chain, one classifier is trained to classify images with variations in the values of the brightness feature, and the other is trained to classify images with variations in the values of the precipitation feature. These classifiers are combined using an OR operator.

For these evaluations, we set resource limits on the python software component (See Figure 7) for evaluating the processing time and memory usage. We assigned a soft limit of one CPU core and a hard limit of four CPU cores on each of the components to mimic the settings of an NVIDIA Jetson TX2 board. Further, to measure the memory usage, we used the psutil [61] cross-platform library.

5.4.1 Comparing Runtime OOD Detection. The false positive and false negative definitions for the $H P, H B, H P B$, and $N R$ test scenes are defined in Table 4. Based on these definitions, the precision and recall of the different detectors for the test scenes are shown in Figure 13. In $H P$ and $H B$ scenes, a single feature (precipitation or brightness) value was varied, and the classifier that was not trained on the representative feature of that scene had low true positives. So, the precision and recall of these detectors are mostly zero. In the HPB scene shown in Figure 13(c), both the features were varied, so a single one-class classifier was not sufficient to identify both the feature variations. However, the one-class classifier chains with an OR logic had higher precision in detecting the feature variations in all these scenes. Similarly, the detection and OOD reasoning capability of the $\beta$-VAE detector could identify both the feature variations.

For the NR scene shown in Figure 13(d), which had a new road segment with the precipitation and brightness values within the training distribution, both the one-class classifiers and their chains raise a false alarm. So, their precision towards detecting variations in these features is low (roughly $1 \%$ ). In contrast, the $\beta$-VAE detector alongside the reasoner was able to identify that the OOD behavior was not because of the precipitation and brightness with a precision of $46 \%$. These results imply that the $\beta$-VAE detector can precisely identify if the features of interest are responsible for the OOD. Whereas a similar reasoning inference cannot be achieved using the other approaches.

5.4.2 Comparing Execution Time and Latency. To emulate a resource constrained setting, we performed the evaluation for execution time and latency on one CPU core.

Execution Time: As discussed earlier, we selected 5 latent variables for detection and one latent variable each for reasoning about the precipitation and brightness features. Two components that mainly contribute to the execution time of the $\beta$-VAE detector are: (1) the time taken by the 

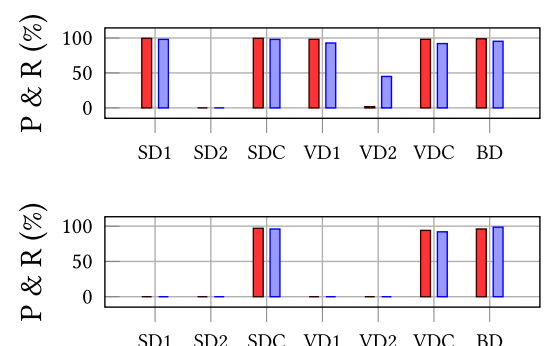
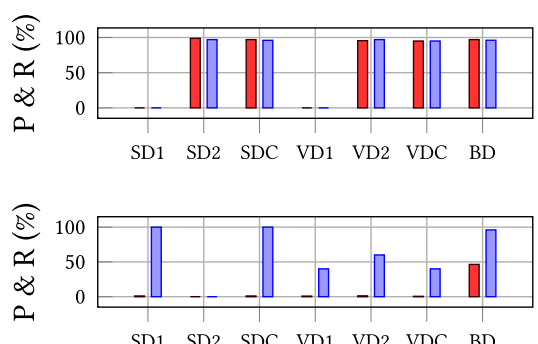

SD1 SD2 SDC VD1 VD2 VDC $\quad$ BD

$P \square R$

Fig. 13. Precision and Recall: Evaluations on different scenes: (a) $H P$ - top left, (b) $H B$ - bottom left, (c) HPB - top right and (d) $N R$ - bottom right. The detectors compared are: SD1 - Deep-SVDD Precipitation detector, $S D 2$ - Deep-SVDD brightness detector, $S D C$ - Deep-SVDD detector chain, VD1 - VAE Precipitation detector, VD2 - VAE brightness detector, VDC - VAE detector chain, BD - $\beta$-VAE detector. These values were collected by running the detectors on each scene for 20 times.

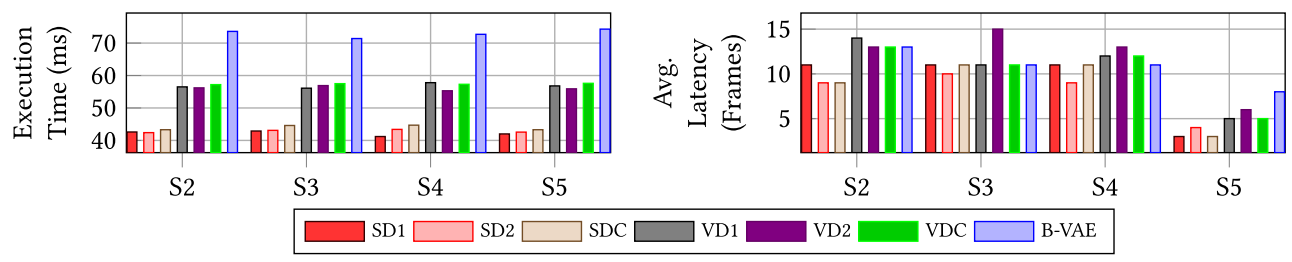

Fig. 14. Timing Analysis: (Left) Execution Time in milliseconds and (Right) Detection latency in number of frames, of the different detectors for the $H P, H B, H P B$, and NR scenes. The detectors are SD1 - Deep-SVDD Precipitation detector, $S D 2$ - Deep-SVDD brightness detector, $S D C$ - Deep-SVDD detector chain, VD1 - VAE Precipitation detector, VD2 - VAE brightness detector, VDC - VAE detector chain, $B D$ - $\beta$-VAE detector. These values were collected by running the detectors on each scene for 20 times.

$\beta$-VAE's encoder to generate the latent variables, and (2) the time taken by ICP and martingale for runtime detection. The average execution time using all 30 latent variables of the $\beta$-VAE was 400 milliseconds, and this was drastically reduced to 74.09 milliseconds when the 5 selected latent variables were used. Also, the reasoner only took about 9 milliseconds as it worked in parallel to the detector.

In comparison, the Deep-SVDD classifiers took an average of 41 milliseconds for detection, and its chain took an average of 43.36 milliseconds, as shown in Figure 14. Also, each of the VAE based reconstruction classifiers took an average of 53 milliseconds for detection, and its chain took an average of 57 milliseconds. The Deep-SVDD and VAE based reconstruction classifiers performed slightly faster than our detector.

Detection Latency: The detection latency in our context is the number of frames between the detection and the occurrence of the OOD. In our approach, the latency is dependent on the size of the martingale window, which is dependent on the CPS dynamics and the sampling period of the system as discussed in Section 3.4. In addition, the selection of the CUSUM threshold also impacts the latency. In these experiments, our AV traveled at a constant speed of $0.5 \mathrm{~m} / \mathrm{s}$, so we used a fixed window size of 20 images. Further, we empirically selected the CUSUM threshold to be 100. With this configuration, the $\beta$-VAE detector had an average latency of 12.6 frames, and the reasoners had an average latency of about 11.5 frames across the 4 test scenes. In comparison, the Deep-SVDD classifiers and their chain had an average latency of 11 frames and 11.21 frames, 
respectively. Also, each of the VAE based reconstruction classifiers and their chain had an average latency of 13 frames and 12.9 frames, respectively.

To summarize, all the approaches had similar detection latency, with the Deep-SVDD classifier performing slightly better. The Deep-SVDD classifier and its chain had slightly shorter latency as compared to our detector. However, the $\beta$-VAE detector had a lower latency compared to the VAE based reconstruction classifier and its chain.

5.4.3 Comparing Memory Usage. Our approach uses a single $\beta$-VAE network to perform both detection and reasoning. Specifically, we only utilize the network's encoder instead of both the encoder and the decoder. The average memory utilization of the $\beta$-VAE detector was $2.49 \mathrm{~GB}$, and the reasoners were $0.23 \mathrm{~GB}$. In comparison, the Deep-SVDD classifiers and their chain utilized an average memory of $3.2 \mathrm{~GB}$ and $6.4 \mathrm{~GB}$, respectively. Also, the VAE based reconstruction classifiers and their chain utilized an average memory of 3.6 GB and 7.2 GB, respectively. The classifier chains utilized higher memory because two DNNss were used for detection.

To summarize, our approach utilizes lesser memory because: (1) it only requires the encoder of a single $\beta$-VAE network for both detection and reasoning; and (2) it utilizes fewer latent variables for detection because it relies on the disentanglement concept. For the AV example, our detector only required 5 latent variables as compared to 1,024 latent variables of the VAE reconstruction classifier and 1,568 activation functions in the embedding layer of the Deep-SVDD classifier.

\subsection{OOD Detection Results from nulmages Dataset}

As the second example, we apply our detection approach on a small fragment of the nuImages [52] dataset. We report the preliminary results of our evaluation in this section.

Dataset Overview: The nuImages dataset is derived from the original nuScenes dataset [7]. The nuImages dataset provides image annotation labels, reduced label imbalance, and a larger number of similar scenes (e.g., a scene with the same road segment but different time-of-day, weather, and pedestrian density values), which makes it suitable for our work. The dataset has 93,000 images collected at $2 \mathrm{~Hz}$, and it has annotations for foreground objects such as vehicles, animals, humans, static obstacles, moving obstacles, and so on. The foreground objects further have additional attributes about the weather, activity of a vehicle, traffic, number of pedestrians, pose of the pedestrians, among others. We demonstrate our OOD detection capability on scenes with different values of traffic and pedestrian density features. For these experiments, we train the detector with different traffic and pedestrian values and then used it to detect images in which either traffic or pedestrian features are absent (OOD condition).

Data Partitioning: The training set $\mathcal{T}$ had images from 4 scenes with varying traffic and pedestrian values. We partitioned $\mathcal{T}$ into two partitions. $P 1$ had images with medium and high pedestrian density values in the range $[1,5]$ and more than 5 , respectively. $P 2$ had images with medium and high traffic values in the range $[1,5]$ and more than 5 , respectively. The test scenes for this evaluation were nominal (Nom) and a pedestrian or traffic (PoT) scene. The Nom scene was in distribution, and it had 30 images in time series captured on a sunny day from a road segment in Singapore city with traffic and pedestrians in the training distribution range. The PoT scene had 70 images in time series captured from a similar road segment, and it either had traffic or pedestrians, but not both together. For most images in this scene, the traffic density took a value in the training range, but the pedestrian value was close to zero. Further, our test scenes were short as it was difficult to find longer image sequences that belonged to a sunny day and similar road segment.

Detector Design: We applied our approach discussed in Section 4 and we used the same $\beta$-VAE network structure as discussed in Section 5.2. Using this set up, we selected a $\beta$-VAE network with hyperparameters $n=30$ and $\beta=1.1$, that resulted in the maximum MIG of 0.0006 . We then identified 

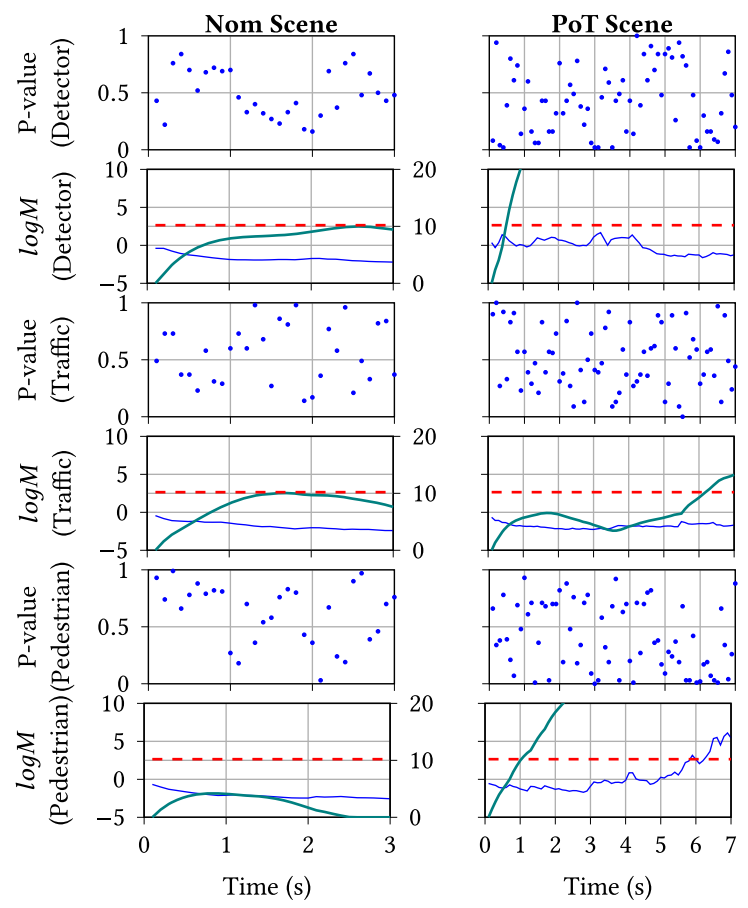

Fig. 15. Runtime OOD detection on nulmages dataset. Performance of the $\beta$-VAE detector for the Nom (nominal) and POT (pedestrian or traffic) scenes of the nulmages experiments. For the martingale plots, the solid blue line represents the log of martingale, the solid green lines represent the CUSUM values, and the dotted red lines represent the threshold $(\tau)$ for CUSUM comparison. Further, the left $y$-axis shows the log of martingale with range $[-5,10]$, and the right $y$-axis shows the CUSUM value with range $[0,20]$. The cusum threshold represented in the red dotted lines are plotted to the right $y$-axis.

the detector latent variable set $\mathcal{L}_{d}$ to be $\left\{L_{0}, L_{7}, L_{9}, L_{22}\right\}$. Further, we selected latent variables $L_{9}$ and $L_{22}$ as the reasoners for the traffic density and pedestrian density partitions, respectively.

OOD Detection: For runtime OOD detection, we used a window size $M=30$ for martingale computation and $\omega=2$ and $\tau=10$ for reasoners and detector CUSUM calculations. The $p$-value and martingale plots for detection and reasoning are shown in Figure 15. The detector Martingale for Nom test scene remained low, and all in-distribution images were detected as in-distribution. The martingale of the traffic density reasoner remained low for most images except for 2 images. We hypothesize the reason for this could be that the vehicle and background blended well that confused the reasoner. The martingale of the pedestrian reasoner remained low throughout the scene. For OOD scene PoT, the detector identified $92 \%$ (65/70) of the images correctly as OOD. However, the first 5 images were incorrectly detected to be in-distribution because of the detection latency of our window-based martingale approach. Also, as the traffic density was mostly zero throughout the scene, the martingale of traffic density reasoner is mostly flat throughout. But, for pedestrian reasoner, all the images were identified as OOD except the first 9 images. The false negatives are primarily because of the complexity of images in a scene and the detection latency. After these images, the martingale increases, and the CUSUM also increase above its threshold. In summary, the reasoners worked reasonably well for these scenes but had a slow martingale growth because of several background attributes like sun glare, trees, and traffic lights.

Challenges: We discuss the several challenges of applying our approach to a real-world dataset. The first challenge is the existence of time series images. Although the nuImages dataset provides 


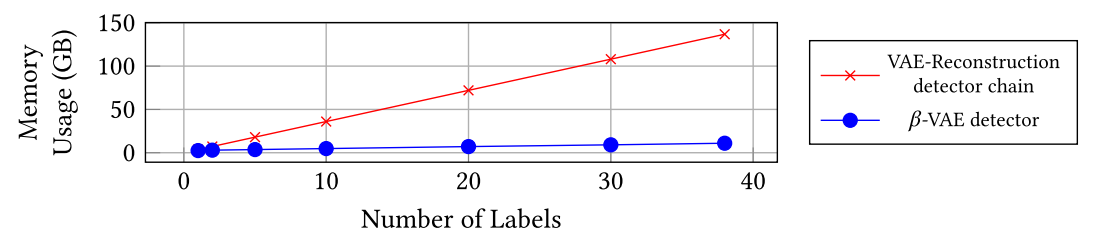

Fig. 16. Memory overhead. Predicted memory usage of a VAE based reconstruction chain and a single $\beta$-VAE detector for the nuScenes images. If a VAE reconstruction detector is used for each of the 38 nuScenes labels, then the memory usage would linearly grow to $136.8 \mathrm{~GB}$. However, a $\beta$-VAE detector based on our approach would only require $10.96 \mathrm{~GB}$. These values were computed based on results in Section 5.4.3.

the notion of a scene, they contain time gaps, especially after partitioning them into train and test the dataset. Second, the labels provided for the dataset images are coarse grain. For example, in nuImages dataset, the semantic label annotations are only limited to high-level foreground objects like pedestrian and cars, and extracting these labels requires significant pre-processing. Another challenge is the complexity of images in real-world datasets. The presence of excess background information such as trees, traffic signals, shadow, reflections, among others, makes the real-world images complex, and it also impacts the information in the latent space. The other challenge is the absence of scenes in which the feature(s) gradually change their values. This makes it difficult to apply our latent variable mapping. Further, finding similar scenes with variations in the specific feature(s) of interest is difficult. For our experiments, we had to perform significant pre-processing to extract short image sequences to perform OOD detection and reasoning.

\subsection{Discussion}

With DNNs being widely used in perception pipelines of automotive CPS, there has been an increased need for OOD detectors that can identify if the operational test image to the DNN is in conformance to the training set. Addressing this problem is challenging because these images have multiple feature labels, and a change in the value of one or more features can cause the image to be OOD. This problem is commonly solved using a multi-chained one-class classifier with each classifier trained on one feature label. However, as shown by our evaluation in Section 5.4, the chain gets computationally expensive with an increased number of image features. So, we have proposed a single $\beta$-VAE detector that is sensitive to variations in multiple features and is computationally inexpensive in comparison to the classifier chains. For example, to perform detection on a real-world automotive dataset like nuScenes with 38 semantic labels, a multi-chained VAE based reconstruction classifier (discussed in our experiments) would require training 38 different VAE DNNs as compared to a single $\beta$-VAE detector that is presented in this work. A memory projection based on the results in Section 5.4.3 is shown in Figure 16. It shows that the multi-chain classifier will need a memory of 136.8 GB. In comparison, our approach requires a single network with one or a few latent variables for detection on each label, and this requires only $10.96 \mathrm{~GB}$ of memory.

Another related problem motivated in this work is the OOD reasoning capability to identify the most responsible feature(s). The reasoning capability is desirable for the system to decide on the mitigation action it must perform. For example, if the detector can identify a high traffic feature to be the cause of the OOD, then the system can switch to an alternate controller with lower autonomy. This reasoning capability cannot be achieved using the state-of-the-art chain of one-class classifiers. But the $\beta$-VAE detector designed and trained using our approach is sensitive to variations in multiple features. We have evaluated this capability of the $\beta$-VAE detector by applying it to several OOD scenes in CARLA simulation (see Figure 11). Especially the $N R$ scene that had a new road segment and background artifacts that are not in the training set. In this 
scene, the multi-chained network identified the scene to be OOD because of the precipitation and brightness features. In comparison, our detector's OOD reasoning capability was able to identify with a precision of $46 \%$ that the cause was not precipitation or brightness.

In addition to the reasoning capability, a detector for a multi-labeled dataset should have minimum sensitivity (defined in Section 2) towards all the feature labels. High minimum sensitivity is needed to detect variations in all the feature labels of the training set images. In our approach, we hypothesize that using the most informative latent variables can provide good minimum sensitivity for the detector towards each feature label. We back this by our results in Table 3, which illustrates that a detector that used the 5 most informative latent variables had a high minimum sensitivity to variations in both the precipitation and brightness features (highlighted in green text in the Table). In comparison, the detectors that used all the latent variables for detection had lower minimum sensitivity, as illustrated in the Table.

Besides identifying if the input images are OOD to the training data, it is necessary to check if they have changed compared to the previous sequence of images in the time series. Such abrupt changes in the current input will increase the system's risk of consequence (e.g., collision) [26], so it is critical to detect them. This problem is necessary to be addressed but has not been given much importance in the OOD detection literature. In this work, we use the detector's latent variables in a moving window based on CUSUM for detecting abrupt changes in the features of the operational test images. We validated our approach across 3 different scenes (see Figure 12) for which our detector could accurately identify feature variations with a short latency of about 11 frames.

Finally, in designing OOD detectors for CPSs, one needs to take into consideration of the system's dynamics. But this is often not given importance in the existing detection approaches. Only recently, Cai et al. [8] have proposed an OOD detection mechanism on time series images that consider the system dynamics. As discussed in Section 4.4, we rely on their ICP and martingale framework approach for runtime detection over a short sliding window of images. The sliding window size needs to be adjusted based on the operational system dynamics like runtime sampling rate and the speed at which the system is traveling.

\section{RELATED WORK}

There has been significant ongoing research to handle the brittleness and susceptibility of DNNs. We have grouped the existing approaches into different classes and briefly discuss them below.

Domain Adaptation involves transferring knowledge between a labeled source domain and an unlabeled target domain [53]. The key idea is to learn domain invariant features of the training data by providing less importance to dataset biases. This is also referred to as transductive transfer learning and is used when the train and the target tasks remain the same while the domains are different. Altering the DNN structure has been one of the approaches that have been used for feature transferability. One such example is the Deep Adaptation Network (DAN) [45] that allows for feature transferability in the task specific layers of DNNs network while reducing domain related information. Biasing the training objective to learn the domain invariant feature(s) has been the other widely adopted approach. For example, Heinze-Deml et al. [28] proposes a conditional variance penalty-based training loss function to learn domain invariant features. Although domain adaptation has been widely used, an assumption on the prior distributions of target domains is restrictive for practical applications like CPSs [73]. Further, negative transfer of knowledge between the source and target domains has been a common problem [78].

Confidence Estimation involves estimating the confidence in the inputs to a DNN. There have been several approaches to estimating confidence. The first approach involves adding a confidence branch at the logits before the softmax layer [13]. The second approach involves using Bayesian Networks for representing uncertainties in the DNNs $[46,51]$. Ensembles of DNNs have been 
another approach used for estimating the confidence in the inputs [22, 41]. All these approaches mostly require making changes to the DNN or will require training model ensembles. Recently, Jha et al. [33] have proposed the attribution based confidence $(A B C)$ metric that does not require access to training data or does not need training model ensembles. It is computed by sampling in the neighborhood of high-dimensional data and then computing a score for its conformance. The metric looks robust, and it does not require access to the training data at runtime, which is an advantage compared to our approach. But the impact of input feature correlations and interactions on the attribution over features require future investigation [67].

Identifying Distribution Shifts involves identifying if the test observation has shifted from the training distribution. Probabilistic classifiers like GAN and VAE have been widely adopted for identifying shifts in the test observations [2, 8, 12, 62]. Our work belongs to this class, and different approaches in this class are discussed in Section 6.1.

In contrast to these approaches, we formulate the problem as a multi-label OOD detection problem, and in addition to identifying an OOD condition, we also find the feature(s) causing it.

\subsection{Probabilistic One-class Classifiers}

6.1.1 Adversarial Networks. GANs have emerged as the leading paradigm in performing unsupervised and semi-supervised tasks like classification and OOD detection [1, 81]. GAN has been shown to outperform classical OOD techniques on benchmark datasets [81]. This network is used along with an adversarial training loss function to perform unsupervised OOD detection [1]. Further, Adversarial Variational Bayes (AVB), a training technique to improve the inference model of VAE [50]. It combines VAEs and GANs to rephrase the maximum-likelihood problem of the VAE as a two player game. Although GAN has performed well in detecting OOD data, there are several problems in using them $[10,75]$. These problems are (1) training complexity because of the instability between the generator and discriminator networks, and (2) mode collapse problem that results in the generator only producing similar samples or, in the worst-case only a single sample.

Adversarial Autoencoder [47] is a probabilistic network that uses the reconstruction capability of an autoencoder along with the adversarial training procedures of a GAN. For example, the reconstruction error is combined with the likelihood in the latent space to perform anomaly detection [4]. Vu, Ha Son et al. [75] have proposed a Dual Adversarial Autoencoder network that uses two autoencoders and discriminators to perform anomaly detection. However, training Adversarial Autoencoders is expensive as it requires training both the autoencoder network and the adversarial network.

6.1.2 Variational Autoencoders (VAE). VAE is the other unsupervised probabilistic generative model that has been used because of its capability to learn latent space of the input data [36]. The latent variables generated by these models are used to learn the correlation between the features of the input data [43]. The VAE based OOD approaches can be classified into two categories.

Reconstruction based techniques use the normalized difference between each point of the input data $(x)$ and the reconstructed data $\left(x^{\prime}\right)$ generated by a VAE. For example, the authors in $[8,59]$ have used the pixel-wise mean square distance between the input image and the reconstructed image as the metric for anomaly detection. As an extension, the reconstruction probability that uses the stochastic latent variables of VAE has been used to compute a probabilistic anomaly score [2]. Although being widely used, this approach can be error-prone when the OOD samples lie on the boundary of the training distribution [12].

Latent space based techniques use distance and density-based metrics on the latent space generated by the encoder of VAE to detect OOD observations. For example, Denouden et al. [12] compute the Mahalanobis distance between the latent distributions of the test image and the mean 
vector of the images in the training set, which is also combined with the reconstruction loss to detect OOD images. The authors in [71] evaluate the latent space with different metrics like Euclidean distance and Bhattacharyya distance for OOD detection. Although being used for OOD detection $[12,71]$, there is a known problem, that the generated latent variables are unstructured, entangled, and lack the ease of understanding [38]. To address this problem, there has been significant research in structuring and disentangling the latent space $[5,9,29,49]$, which is discussed in the following section.

\subsection{Disentangling Latent Representations}

Learning a disentangled latent space have been shown to be beneficial for several tasks like pose invariant face recognition [55, 69], video predictions [30], and anomaly detection [76]. While there are several approaches to learning a disentangled latent space [5], of particular interest to this work are approaches that use the VAE structure. Recently, variants of a VAE like FactorVAE [35], $\beta$-VAE [29], and $\beta$-TCVAE [9] have been used. Among these, $\beta$-VAE has been widely used because it provides a single $\beta$ gating hyperparameter that can be used control the latent space disentanglement [29]. Mathieu, Emile et al. [49] illustrates that a $\beta$-VAE generates a lower overlap of the latent variables as compared to the original VAE, and illustrates how different values of $\beta$ impacts the latent variable overlap. Further, Locatello et al. [44] have illustrated with experiments that network inductive biases are necessary to achieve unsupervised disentanglement.

In recent years $\beta$-VAE is used for OOD detection tasks because of its ability to generate a disentangled latent space. In our previous work [66], we have used the tuning capability of a $\beta$-VAE to make it sensitive to different image features (e.g., traffic density, pedestrians) and then used it to detect changes in those features. Graydon et al. [25] have used the latent variables generated by a $\beta$-VAE along with Gaussian mixture models to identify OOD on cancer datasets. A combination of the reconstruction error and the distances among the latent variables of a $\beta$-VAE is used as the anomaly score [42]. Further, a $\beta$-VAE is shown to perform better for anomaly detection on MRI datasets as compared to a classical VAE [82]. However, these approaches either randomly select the $\beta$ values or manually tune them, which can be expensive and time consuming. In contrast, our work provides a Bayesian Optimization based heuristic to select the $\beta$ value.

Further, to measure the level of disentanglement, several methods and metrics are available. Visualization of reconstructions by inspecting latent traversals has been a simple method that has been widely used. But visualization alone is not sufficient, as discussed in Section 1. So, several qualitative and quantitative metrics have been proposed for this task. A classifier based supervised learning metrics $[24,29,35]$ have been designed when all the underlying generative factors of an image are known. For example, the BetaVAE metric [29] builds a linear classifier that predicts the index of a fixed factor with variations. However, this metric has a drawback of axis alignment, and this is overcome by the FactorVAE metric [35] by using a majority vote classifier. Also, these metrics require building and tuning the sensitivity of the classifiers, which is difficult to design and train. This problem is overcome by the Mutual Information Gap [9] metric, which is a quantitative metric based on the mutual information between the features and latent variables. In this work, we have used MIG to measure the level of latent space disentanglement.

\section{CONCLUSIONS AND FUTURE WORK}

In this article, we proposed a design approach to generate a partially disentangled latent space and learn an approximate mapping between the latent variables and features for OOD detection and reasoning. To generate the disentangled latent space, we use a $\beta$-VAE network and tune its hyperparameter $(n, \beta)$ using a Bayesian Optimization heuristic. Next, we perform a latent variable mapping to identify the most informative latent variables for detection and identify latent 
variable(s) which are sensitive to specific features and use them for reasoning the OOD problem. We evaluated our approach using an AV example in the CARLA simulation and illustrated the preliminary results from the nuImages dataset. Our evaluation has shown that the detector designed using our approach has good robustness, minimum sensitivity, and low execution time. The detector could also detect OOD images and identify the most likely feature(s) causing it.

Future extensions and applications of the proposed approach include: (1) improving the latent variable mapping heuristic to perform robust correspondence between the features and latent variables, (2) explore alternate metrics such as Wasserstein distance for latent variable mapping, (3) apply the approach to real-world datasets and research CPS testbeds like DeepNNCar [57], and (4) use the anomaly detection results to perform higher-level decision making such as controller selection or enactment of contingency plans.

\section{ACKNOWLEDGMENTS}

We thank the anonymous reviewers of our journal for their insightful comments and valuable suggestions.

\section{REFERENCES}

[1] Samet Akcay, Amir Atapour-Abarghouei, and Toby P. Breckon. 2019. GANomaly: Semi-supervised anomaly detection via adversarial training. In Proceedings of the Computer Vision - ACCV 2018. C. V. Jawahar, Hongdong Li, Greg Mori, and Konrad Schindler (Eds.). Springer International Publishing, Cham, 622-637.

[2] Jinwon An and Sungzoon Cho. 2015. Variational autoencoder based anomaly detection using reconstruction probability. Special Lecture on IE 2, 1 (2015), 1-18.

[3] Michèle Basseville and Igor V. Nikiforov. 1993. Detection of Abrupt Changes: Theory and Application, Vol. 104. prentice Hall Englewood Cliffs.

[4] Laura Beggel, Michael Pfeiffer, and Bernd Bischl. 2019. Robust anomaly detection in images using adversarial autoencoders. In Proceedings of the foint European Conference on Machine Learning and Knowledge Discovery in Databases. Springer, 206-222.

[5] Yoshua Bengio, Aaron Courville, and Pascal Vincent. 2013. Representation learning: A review and new perspectives. IEEE Transactions on Pattern Analysis and Machine Intelligence 35, 8 (2013), 1798-1828.

[6] Mariusz Bojarski, Davide Del Testa, Daniel Dworakowski, Bernhard Firner, Beat Flepp, Prasoon Goyal, Lawrence D. Jackel, Mathew Monfort, Urs Muller, Jiakai Zhang, Xin Zhang, Jake Zhao, and Karol Zieba. 2016. End to end learning for self-driving cars. arXiv:1604.07316. Retrieved from https://arxiv.org/abs/1604.07316.

[7] Holger Caesar, Varun Bankiti, Alex H. Lang, Sourabh Vora, Venice Erin Liong, Qiang Xu, Anush Krishnan, Yu Pan, Giancarlo Baldan, and Oscar Beijbom. 2020. nuscenes: A multimodal dataset for autonomous driving. In Proceedings of the IEEE/CVF Conference on Computer Vision and Pattern Recognition. 11621-11631.

[8] Feiyang Cai and Xenofon Koutsoukos. 2020. Real-time out-of-distribution detection in learning-enabled cyberphysical systems. In Proceedings of the 2020 ACM/IEEE 11th International Conference on Cyber-Physical Systems. IEEE, $174-183$

[9] Tian Qi Chen, Xuechen Li, Roger B. Grosse, and David K. Duvenaud. 2018. Isolating sources of disentanglement in variational autoencoders. In Proceedings of the Advances in Neural Information Processing Systems. 2610-2620.

[10] Antonia Creswell, Tom White, Vincent Dumoulin, Kai Arulkumaran, Biswa Sengupta, and Anil A. Bharath. 2018. Generative adversarial networks: An overview. IEEE Signal Processing Magazine 35, 1 (2018), 53-65.

[11] Igor Dejanović, Renata Vaderna, Gordana Milosavljević, and Željko Vuković. 2017. TextX: A python tool for domainspecific languages implementation. Knowledge-Based Systems 115 (2017), 1-4.

[12] Taylor Denouden, Rick Salay, Krzysztof Czarnecki, Vahdat Abdelzad, Buu Phan, and Sachin Vernekar. 2018. Improving reconstruction autoencoder out-of-distribution detection with Mahalanobis distance. arXiv:1812.02765. Retrieved from https://arxiv.org/abs/1812.02765.

[13] Terrance DeVries and Graham W. Taylor. 2018. Learning confidence for out-of-distribution detection in neural networks. arXiv:1802.04865. Retrieved from https://arxiv.org/abs/1802.04865.

[14] Alexey Dosovitskiy, German Ros, Felipe Codevilla, Antonio Lopez, and Vladlen Koltun. 2017. CARLA: An open urban driving simulator. Conference on robot learning.

[15] Abhishek Dubey, Gabor Karsai, and Nagabhushan Mahadevan. 2011. Model-based software health management for real-time systems. In Proceedings of the 2011 Aerospace Conference. IEEE, 1-18. 
[16] Sheri Edwards. 2008. Elements of Information Theory, Thomas M. Cover, Joy A. Thomas, John Wiley \& Sons, Inc. (2006). 18 pages.

[17] İkbal Eski and SCahin Yildirim. 2014. Design of neural network control system for controlling trajectory of autonomous underwater vehicles. International Journal of Advanced Robotic Systems 11, 1 (2014), 7.

[18] Valentina Fedorova, Alex Gammerman, Ilia Nouretdinov, and Vladimir Vovk. 2012. Plug-in martingales for testing exchangeability on-line. arXiv:1204.3251. Retrieved from https://arxiv.org/abs/1204.3251.

[19] [Online] foretellix. [n.d.]. Open M-SDL. Retrieved May 2020 from https://www.foretellix.com/open-language/.

[20] Daniel J. Fremont, Tommaso Dreossi, Shromona Ghosh, Xiangyu Yue, Alberto L. Sangiovanni-Vincentelli, and Sanjit A. Seshia. 2019. Scenic: A language for scenario specification and scene generation. In Proceedings of the 40th ACM SIGPLAN Conference on Programming Language Design and Implementation. 63-78.

[21] Jacob R. Gardner, Matt J. Kusner, Zhixiang Eddie Xu, Kilian Q. Weinberger, and John P. Cunningham. 2014. Bayesian optimization with inequality constraints. In Proceedings of the ICML, Vol. 2014. 937-945.

[22] Yonatan Geifman, Guy Uziel, and Ran El-Yaniv. 2018. Bias-reduced uncertainty estimation for deep neural classifiers. arXiv:1805.08206. Retrieved from https://arxiv.org/abs/1805.08206.

[23] Ian Goodfellow, Jean Pouget-Abadie, Mehdi Mirza, Bing Xu, David Warde-Farley, Sherjil Ozair, Aaron Courville, and Yoshua Bengio. 2014. Generative adversarial nets. In Proceedings of the Advances in Neural Information Processing Systems. 2672-2680.

[24] Will Grathwohl and Aaron Wilson. 2016. Disentangling space and time in video with hierarchical variational autoencoders. arXiv:1612.04440. Retrieved from https://arxiv.org/abs/1612.04440.

[25] Tucker Graydon and Ferat Sahin. 2018. Novelty detection and analysis with a Beta-VAE network. In Proceedings of the 2018 IEEE International Conference on Systems, Man, and Cybernetics. IEEE, 2687-2691.

[26] C. Hartsell, S. Ramakrishna, A. Dubey, D. Stojcsics, N. Mahadevan, and G. Karsai. 2021. ReSonAte: A runtime risk assessment framework for autonomous systems. In Proceedings of the 20212021 International Symposium on Software Engineering for Adaptive and Self-Managing Systems. IEEE Computer Society, 118-129. DOI : https://doi.org/10.1109/ SEAMS51251.2021.00025

[27] Trevor Hastie, Robert Tibshirani, and Jerome Friedman. 2009. The Elements of Statistical Learning: Data Mining, Inference, and Prediction. Springer Science \& Business Media.

[28] Christina Heinze-Deml and Nicolai Meinshausen. 2021. Conditional variance penalties and domain shift robustness. Machine Learning 110, 2 (2021), 303-348.

[29] Irina Higgins, Loic Matthey, Arka Pal, Christopher Burgess, X-avier Glorot, Matthew Botvinick, Shakir Mohamed, and Alexan-der Lerchner. 2016. Beta-VAE: Learning basic visual concepts with a constrained variational framework. In Proceedings of the ICLR17 (2016).

[30] Jun-Ting Hsieh, Bingbin Liu, De-An Huang, Li F. Fei-Fei, and Juan Carlos Niebles. 2018. Learning to decompose and disentangle representations for video prediction. In Proceedings of the Advances in Neural Information Processing Systems. 517-526.

[31] Frank Hutter, Lars Kotthoff, and Joaquin Vanschoren. 2019. Automated Machine Learning: Methods, Systems, Challenges. Springer Nature.

[32] Marek Jakab, Lukas Hudec, and Wanda Benesova. 2020. Partial disentanglement of hierarchical variational autoencoder for texture synthesis. IET Computer Vision 14, 8 (2020), 564-574.

[33] Susmit Jha, Sunny Raj, Steven Fernandes, Sumit Kumar Jha, Somesh Jha, Brian Jalaian, Gunjan Verma, and Ananthram Swami. 2019. Attribution-based confidence metric for deep neural networks. In Proceedings of the 33rd International Conference on Neural Information Processing Systems.

[34] Donald R. Jones, Matthias Schonlau, and William J. Welch. 1998. Efficient global optimization of expensive black-box functions. Journal of Global Optimization 13, 4 (1998), 455-492.

[35] Hyunjik Kim and Andriy Mnih. 2018. Disentangling by factorising. In Proceedings of the International Conference on Machine Learning. PMLR, 2649-2658.

[36] Diederik P. Kingma and Max Welling. 2013. Auto-encoding variational bayes. arXiv:1312.6114. Retrieved from https: //arxiv.org/abs/1312.6114

[37] Diederik P. Kingma and Max Welling. 2019. An introduction to variational autoencoders. arXiv:1906.02691 (2019). Retrieved from https://arxiv.org/abs/1906.02691.

[38] Jack Klys, Jake Snell, and Richard Zemel. 2018. Learning latent subspaces in variational autoencoders. In Proceedings of the Advances in Neural Information Processing Systems. 6444-6454.

[39] ByungSoo Ko, Ho-Jin Choi, Chansol Hong, Jong-Hwan Kim, Oh Chul Kwon, and Chang D. Yoo. 2017. Neural networkbased autonomous navigation for a homecare mobile robot. In Proceedings of the 2017 IEEE International Conference on Big Data and Smart Computing. IEEE, 403-406.

[40] Puneet Kohli and Anjali Chadha. 2019. Enabling pedestrian safety using computer vision techniques: A case study of the 2018 uber inc. self-driving car crash. In Proceedings of the Future of Information and Communication Conference. Springer, 261-279. 
[41] Balaji Lakshminarayanan, Alexander Pritzel, and Charles Blundell. 2017. Simple and scalable predictive uncertainty estimation using deep ensembles. In Proceedings of the 31st International Conference on Neural Information Processing Systems. Curran Associates Inc., Red Hook, NY, USA, 6405-6416.

[42] Xiaoyan Li. 2020. Anomaly Detection Based on Disentangled Representation Learning. Ph.D. Dissertation. Université d'Ottawa/University of Ottawa.

[43] Yang Liu, Eunice Jun, Qisheng Li, and Jeffrey Heer. 2019. Latent space cartography: Visual analysis of vector space embeddings. In Proceedings of the Computer Graphics Forum, Vol. 38. Wiley Online Library, 67-78.

[44] Francesco Locatello, Stefan Bauer, Mario Lucic, Gunnar Raetsch, Sylvain Gelly, Bernhard Schölkopf, and Olivier Bachem. 2019. Challenging common assumptions in the unsupervised learning of disentangled representations. In Proceedings of the International Conference on Machine Learning. PMLR, 4114-4124.

[45] Mingsheng Long, Yue Cao, Jianmin Wang, and Michael Jordan. 2015. Learning transferable features with deep adaptation networks. In Proceedings of the International Conference on Machine Learning. PMLR, 97-105.

[46] David J. C. MacKay. 1992. A practical Bayesian framework for backpropagation networks. Neural Computation 4, 3 (1992), 448-472.

[47] Alireza Makhzani, Jonathon Shlens, Navdeep Jaitly, Ian Goodfellow, and Brendan Frey. 2015. Adversarial autoencoders. arXiv:1511.05644. Retrieved from https://arxiv.org/abs/1511.05644.

[48] Francisco J. Martinez-Estudillo, Pedro Antonio Gutiérrez, César Hervás, and Juan Carlos Fernández. 2008. Evolutionary learning by a sensitivity-accuracy approach for multi-class problems. In Proceedings of the 2008 IEEE Congress on Evolutionary Computation (IEEE World Congress on Computational Intelligence). IEEE, 1581-1588.

[49] Emile Mathieu, Tom Rainforth, Nana Siddharth, and Yee Whye Teh. 2019. Disentangling disentanglement in variational autoencoders. In Proceedings of the International Conference on Machine Learning. PMLR, 4402-4412.

[50] Lars Mescheder, Sebastian Nowozin, and Andreas Geiger. 2017. Adversarial variational bayes: Unifying variational autoencoders and generative adversarial networks. In Proceedings of the International Conference on Machine Learning. PMLR, 2391-2400.

[51] Radford M. Neal. 2012. Bayesian Learning for Neural Networks, Vol. 118. Springer Science \& Business Media.

[52] [Online] nuscenes. [n.d.]. nuImage dataset. Retrieved June 2020 from https://www.nuscenes.org/nuimages.

[53] Sinno Jialin Pan and Qiang Yang. 2009. A survey on transfer learning. IEEE Transactions on Knowledge and Data Engineering 22, 10 (2009), 1345-1359.

[54] Kexin Pei, Yinzhi Cao, Junfeng Yang, and Suman Jana. 2017. DeepXplore: Automated whitebox testing of deep learning systems. In Proceedings of the 26th Symposium on Operating Systems Principles. ACM, 1-18.

[55] Xi Peng, Xiang Yu, Kihyuk Sohn, Dimitris N. Metaxas, and Manmohan Chandraker. 2017. Reconstruction-based disentanglement for pose-invariant face recognition. In Proceedings of the IEEE International Conference on Computer Vision. 1623-1632.

[56] Dean A. Pomerleau. 1989. ALVINN: An autonomous land vehicle in a neural network. In Proceedings of the Advances in Neural Information Processing Systems. 305-313.

[57] Shreyas Ramakrishna, Charles Harstell, Matthew P. Burruss, Gabor Karsai, and Abhishek Dubey. 2020. Dynamicweighted simplex strategy for learning enabled cyber physical systems. Fournal of Systems Architecture 111 (2020), 101760. DOI : https://doi.org/10.1016/j.sysarc.2020.101760

[58] Jesse Read, Bernhard Pfahringer, Geoff Holmes, and Eibe Frank. 2011. Classifier chains for multi-label classification. Machine Learning 85, 3 (2011), 333.

[59] Charles Richter and Nicholas Roy. 2017. Safe Visual Navigation via Deep Learning and Novelty Detection. Robotics: Science and Systems Foundation (2017).

[60] Haakon Ringberg, Augustin Soule, Jennifer Rexford, and Christophe Diot. 2007. Sensitivity of PCA for traffic anomaly detection. In Proceedings of the 2007 ACM SIGMETRICS International Conference on Measurement and Modeling of Computer Systems. 109-120.

[61] Giampaolo Rodola. 2016. Psutil package: A cross-platform library for retrieving information on running processes and system utilization. Retrieved Jan 2020 from https://psutil.readthedocs.io/en/latest/.

[62] Lukas Ruff, Robert Vandermeulen, Nico Goernitz, Lucas Deecke, Shoaib Ahmed Siddiqui, Alexander Binder, Emmanuel Müller, and Marius Kloft. 2018. Deep one-class classification. In Proceedings of the International Conference on Machine Learning. 4393-4402.

[63] Bernhard Schölkopf, John C. Platt, John Shawe-Taylor, Alex J. Smola, and Robert C. Williamson. 2001. Estimating the support of a high-dimensional distribution. Neural Computation 13, 7 (2001), 1443-1471.

[64] D. Seto, B. H. Krogh, L. Sha, and A. Chutinan. 1998. The simplex architecture for safe on-line control system upgrades. In Proceedings of the American Control Conference, Vol. 6. AMERICAN AUTOMATIC CONTROL COUNCIL, 3504-3508.

[65] Jasper Snoek, Hugo Larochelle, and Ryan P. Adams. 2012. Practical Bayesian optimization of machine learning algorithms. In Proceedings of the Advances in Neural Information Processing Systems. 2951-2959. 
[66] V. Sundar, S. Ramakrishna, Z. Rahiminasab, A. Easwaran, and A. Dubey. 2020. Out-of-distribution detection in multilabel datasets using latent space of $\hat{\mathrm{I}}^{2}$-VAE. In Proceedings of the 2020 IEEE Security and Privacy Workshops. IEEE Computer Society, 250-255. DOI : https://doi.org/10.1109/SPW50608.2020.00057

[67] Mukund Sundararajan, Ankur Taly, and Qiqi Yan. 2017. Axiomatic attribution for deep networks. In Proceedings of the International Conference on Machine Learning. PMLR, 3319-3328.

[68] Yuchi Tian, Kexin Pei, Suman Jana, and Baishakhi Ray. 2018. DeepTest: Automated testing of deep-neural-networkdriven autonomous cars. In Proceedings of the 40th International Conference on Software Engineering. ACM, 303-314.

[69] Luan Tran, Xi Yin, and Xiaoming Liu. 2017. Disentangled representation learning gan for pose-invariant face recognition. In Proceedings of the IEEE Conference on Computer Vision and Pattern Recognition. 1415-1424.

[70] Grigorios Tsoumakas, Ioannis Katakis, and Ioannis Vlahavas. 2009. Mining multi-label data. In Proceedings of the Data Mining and Knowledge Discovery Handbook. Springer, 667-685.

[71] Aleksei Vasilev, Vladimir Golkov, Marc Meissner, Ilona Lipp, Eleonora Sgarlata, Valentina Tomassini, Derek K. Jones, and Daniel Cremers. 2020. q-space novelty detection with variational autoencoders. In Proceedings of the Computational Diffusion MRI. Elisenda Bonet-Carne, Jana Hutter, Marco Palombo, Marco Pizzolato, Farshid Sepehrband, and Fan Zhang (Eds.). Springer International Publishing, Cham, 113-124.

[72] Bill Vlasic and Neal E. Boudette. 2016. 'Self-driving tesla was involved in fatal crash,' US says. New York Times 302016 (2016).

[73] Riccardo Volpi, Hongseok Namkoong, Ozan Sener, John C. Duchi, Vittorio Murino, and Silvio Savarese. 2018. Generalizing to unseen domains via adversarial data augmentation. In Proceedings of the NeurIPS. 5339-5349. Retrieved from http://papers.nips.cc/paper/7779-generalizing-to-unseen-domains-via-adversarial-data-augmentation.

[74] Vladimir Vovk, Alex Gammerman, and Glenn Shafer. 2005. Algorithmic Learning in a Random World. Springer Science \& Business Media.

[75] Ha Son Vu, Daisuke Ueta, Kiyoshi Hashimoto, Kazuki Maeno, Sugiri Pranata, and Sheng Mei Shen. 2019. Anomaly detection with adversarial dual autoencoders. arXiv:1902.06924. Retrieved from https://arxiv.org/abs/1701.00133.

[76] Shuo Wang, Tianle Chen, Shangyu Chen, C. Rudolph, S. Nepal, and M. Grobler. 2020. OIAD: One-for-all image anomaly detection with disentanglement learning. In Proceedings of the 2020 International foint Conference on Neural Networks (2020), 1-8.

[77] Shijin Wang, Jianbo Yu, Edzel Lapira, and Jay Lee. 2013. A modified support vector data description based novelty detection approach for machinery components. Applied Soft Computing 13, 2 (2013), 1193-1205.

[78] Zirui Wang, Zihang Dai, Barnabás Póczos, and Jaime Carbonell. 2019. Characterizing and avoiding negative transfer. In Proceedings of the IEEE/CVF Conference on Computer Vision and Pattern Recognition. 11293-11302.

[79] BP Welford. 1962. Note on a method for calculating corrected sums of squares and products. Technometrics 4, 3 (1962), 419-420.

[80] Chih-Kuan Yeh, Wei-Chieh Wu, Wei-Jen Ko, and Yu-Chiang Frank Wang. 2017. Learning deep latent space for multilabel classification. In Proceedings of the 31 AAAI Conference on Artificial Intelligence.

[81] Houssam Zenati, Chuan-Sheng Foo, Bruno Lecouat, G. Manek, and V. Chandrasekhar. 2018. Efficient GAN-based anomaly detection. arXiv:1802.06222. Retrieved from https://arxiv.org/abs/1802.06222.

[82] Leixin Zhou, Wenxiang Deng, and Xiaodong Wu. 2020. Unsupervised anomaly localization using VAE and beta-VAE. arXiv:2005.10686. Retrieved from https://arxiv.org/abs/2005.10686.

Received August 2020; revised April 2021; accepted August 2021 